\title{
An OpenFOAM-Based Evaluation of PANS Methodology in Conjunction with Non-Linear Eddy Viscosity: Flow Past a Heated Cylinder
}

\author{
S. Saroha ${ }^{1}$, K. Chakraborty ${ }^{1}$, S. S. Sinha ${ }^{1 \dagger}$ and S. Lakshmipathy ${ }^{2}$ \\ ${ }^{1}$ Applied Mechanics Department, IIT Delhi, 110016, India \\ ${ }^{2}$ Gexcon AS, Bergen, 5072, Norway \\ $\dagger$ Corresponding Author Email: sawan@am.iitd.ac.in
}

(Received August 26, 2019; accepted March 2, 2020)

\begin{abstract}
We evaluate the partially-averaged Navier-Stokes (PANS) methodology of turbulence computations by including non-linear eddy viscosity based closures for both turbulent stresses and thermal flux. We extract the filtered PANS version of the Shih's quadratic model (originally proposed for the Reynolds averaged NavierStokes (RANS) paradigm) for arriving at a PANS closure model for the turbulent stress tensor. The unclosed thermal flux process is modeled using the gradient diffusion hypothesis, wherein we sensitize the coefficient of diffusion to the presence of non-linear stresses in the formulation. The resulting methodology is evaluated by simulating flow past a heated square cylinder. Evaluations are performed in terms of both hydrodynamic variables and heat transfer characteristics. We find that the non-linear PANS methodology shows improved results in terms of hydrodynamic quantities (coefficient of drag, pressure, velocity profiles, and high-order statistics). While the predictions of the heat transfer rate on the front face of the cylinder are similar in the linear and the non-linear PANS methodologies, in the wake region and parts of the lateral wall where shear layer detachment takes place, the non-linear PANS methodology shows improved results.
\end{abstract}

Keywords: Non-linear eddy viscosity closure; Scale resolving simulations; Turbulent heat transfer; Separated flows, PANS.

\section{INTRODUCTION}

Accurate numerical predictions of heat transfer rate at a solid-fluid interface is crucial for arriving at efficient de-signs of many engineering systems like heat exchangers, cooling towers, electronic circuit boards, aircraft engines and power turbines. Many engineering applications involve forced convective heat transfer process wherein the flow field is turbulent as well as separated from the solid surface giving rise to unsteadiness across all scales of motion. These features (turbulence and flow separation) exacerbate the complications in the flow field making it quite challenging for the computational fluid dynamics (CFD) methodologies to capture even the most essential flow physics.

The Reynolds-averaged Navier-Stokes (RANS) equation and direct numerical simulations (DNS) are two extreme CFD approaches that can be employed for prediction of turbulent flows with heat transfer. DNS is expected to resolve all scales of motion on an adequately fine grid, which could be quite accurate but has a prohibitive computational demand. The Reynolds-averaged Navier-Stokes (RANS) methodology requires less computational resources, but requires high-fidelity turbulence closure models. Typically, in separated turbulent flows, RANS - because of its indiscriminate averaging of all scales of motion and a consequent over-reliance on turbulence closure models, yields inaccurate predictions. In between the extremes of the RANS and the DNS methods, other computational paradigms exist such as large eddy simulations (LES) and the bridging methods of turbulence computations (Schiestel and Dejoan 2005; Girimaji 2006), which can be collectively called the scale-resolving simulations. Such methodologies use the filtering approach, wherein scales of motion larger than the filter are resolved, whereas those scales which are smaller than the filter cutoff are modeled. The computational demand of LES is less than that of the DNS, however, it is still large enough to be prohibitive for many users. A bridging method, on the other hand, allows the user to place the filter cutoff anywhere between the largest to the dissipative scales of motion commensurating with the computational 
resource that the user can afford. In this work we focus on one such bridging method called the partially-averaged Navier-Stokes (PANS) method (Girimaji 2006).

In recent past, the PANS methodology has been mostly employed in simulating hydrodynamic aspects of various kinds of flow fields (Girimaji 2006; Abdol-Hamid and Girimaji 2004; Jeong and Girimaji 2010; Lakshmipathy and Girimaji 2010; Rao et al. 2018; Saroha et al. 2018; Basara and Girimaji 2018; Pereira et al. 2018b). Ranjan and Dewan (2015) has employed PANS for simulating flow past a heated square cylinder. However, all these works have employed conventional linear Boussinesq approximation (linear eddy viscosity closures) for the unclosed turbulent stress as well for the unclosed thermal flux. In these studies turbulent stresses in the momentum equation are assumed to be proportional to the resolved strainrate. Similarly, following the gradient diffusion hypothesis, the turbulent thermal flux (the unclosed term in the filtered energy equation) is assumed to be proportional to the filtered temperature gradient with the diffusion coefficient dependent on the local eddy viscosity. In separated flows, however, these simple linear models may not be adequate (see Speziale (1987), Shih et al. (1993), Saroha et al. (2018)). Indeed Chesnakas et al. (1997), based on their experimental measurements of separated flows past a prolate spheroid, reported significant misalignment between the strain-rate tensor and the Reynolds stress tensor and highlighted the prospect of improving CFD predictions by using a non-linear constitutive relationship. Even though PANS inherently is capable of allowing more scales of motion to be resolved compared to RANS, the limitations of a linear constitutive relationship is bound to constrain the performance of PANS like it does for RANS. Thus, it is plausible to explore further improvement in the performance of PANS methodology by using non-linear eddy viscosity closure models (NLEVM).

In our recent work (Saroha et al. 2019), we have presented model extraction and some evaluations of PANS methodology using a non-linear closure for turbulent stresses. Subsequent to model extraction, we performed the so-called fixed-point analysis, wherein the essential energetics of the closure model is examined in the absence of transport processes in the governing equations. We clearly demonstrated that with a reduction in the cut-off determining filter, the non-linear PANS methodology indeed releases more scale of motion even with the presence of non-linear stresses. Further, we examined the influence of non-linear eddy viscosity on the extent of misalignment between the turbulent stress tensor with the resolved strain-rate and its consequent allowance for the resolved rotation-rate tensor to influence the unclosed stress tensor. In the same work (Saroha et al. 2019), some preliminary evaluations were performed for the case of flow past a square cylinder as well. However, the computational grid employed therein used standard wall functions and no heat transfer aspects were considered.

Thus, the goal of this work is to extend the PANS methodology in conjugation with the non-linear eddy viscosity closure to simulate separated flows with heat transfer and compare its performance with the conventional PANS methodology, which uses the Boussinesq's linear eddy viscosity model. We would like to highlight that, unlike our previous work (Saroha et al. 2019), in this work all simulations are performed using superior wallresolving grids along with the inclusion of lowReynolds number near-wall damping effects in the closure model. Thus, even from the point of view of purely hydrodynamic quantities, the evaluations performed in this study can be deemed to be more comprehensive than what we presented earlier in (Saroha et al. 2019).

Specifically, we perform the following tasks in this study:

1. Using the quadratic RANS model of Shih et al.(1993), we extract the PANS version of the closure model for turbulent stress tensor in the filtered momentum equation.

2. We model the unclosed thermal flux process incumbent in the filtered temperature equation using the gradient diffusion hypothesis, wherein we appropriately sensitize the coefficient of eddy diffusion to the presence of non-linear stresses in the formulation.

3. We evaluate the non-linear eddy viscosity based PANS formulation in flow past a heated square cylinder at Reynolds number of 21,400.

4. To clearly identify the improvements of the non-linear PANS methodology, we perform simulations using the conventional linear eddy viscosity based PANS methodology as well.

The flow past a heated cylinder has been used by several researchers in the past to test new turbulence closure models and numerical schemes alike Franke and Rodi (1993), Rodi (1997), Kimura and Hosoda (2003) , Frendi et al. (2006), Aus der Wiesche (2007), Ayyappan and Vengadesan (2008), Liu (2010), Moulai et al.(2016). Despite having a simple geometry, the flow past a square cylinder involves several complex flow phenomena like extended stagnation-like region, shear layer detachment, instabilities leading to turbulence and unsteady vortex shedding- making it an excellent test case. Recently, Pereira et al. (2018a) have highlighted the importance of addressing these challenges in context of scale-resolving simulations. Our simulations results are compared against the experimental measurements of Igarashi (1985) (for heat transfer rates), Lyn et al. (1995) and Igarashi (1984) (for hydrodynamics variables). Further, recent DNS results of Trias et al. (2015) are also included for reference.

The authors would like to make it clear that the intent of this work is not to claim that employing a non-linear eddy viscosity in conjugation with PANS would necessarily result into the "best" predictions 
of flow variables. The scope of the present work is limited to exploring and evaluating the consequences, when given the same two-equation model framework (in this work the k- $\varepsilon$ framework), closure is allowed to switch from the conventional linear eddy viscosity closure to a nonlinear eddy viscosity closure. Further, the authors acknowledge that there are several non-linear RANS closures available in literature (Argyropoulos and Markatos 2015; Hellsten and Wallin 2009). Our choice of the Shih's quadratic model is governed by: (i) Its improved performance in several flow fields (Shih et al. 1997; Ishihara and Hibi 2002; Yang et al. 2005; Colombo et al. 2008), and (ii) Its availability in the OpenFOAM library (which the authors employ for this study). Indeed, the authors believe that this study will motivate more users to further explore and evaluate the integration of PANS methodology with other nonlinear and even more explicit algebraic Reynolds stress models (EARSMs) as well (Wallin and Johansson 2000; Girimaji 1997). GitHub link for source code is provided here: https://github.com/sagarsaroha18/OpenFOAM

This paper has been organized into six sections. In section 2, we provide a brief overview of the PANS method. In this section, we first discuss the PANS version of the non-linear eddy viscosity closure for unclosed stresses.

Subsequently, we present our modelling strategy for the unclosed thermal flux process in the temperature equation. In section 3, we present details about our computational set up and our plan of study. Descriptions of the quantities of interest, time step convergence and grid convergence studies are included in section 4 . In section 5, we compare the performance of the non-linear and linear closures of PANS methodologies. Section 6 concludes the paper with a summary.

\section{PARTIALly-AVERAGED NAVIER- STOKES EQUATIONS (PANS)}

We start with the instantaneous momentum, continuity and temperature equation set (1) applicable for a constant density flow field. We assume that this flow field is at a high Froude number and at a low Grashof number. Hence, the effects of buoyancy and body forces are neglected.

$$
\begin{aligned}
& \frac{\partial V_{i}}{\partial t}+V_{j} \frac{\partial V_{i}}{\partial x_{j}}=-\frac{1}{\rho} \frac{\partial p}{\partial x_{i}}+\frac{\partial}{\partial x_{j}}\left(v \frac{\partial V_{i}}{\partial x_{j}}\right) \\
& \frac{\partial V_{i}}{\partial x_{i}}=0 ; \frac{\partial T}{\partial t}+V_{j} \frac{\partial T}{\partial x_{j}}=\frac{\partial}{\partial x_{j}}\left(\frac{v}{\operatorname{Pr}} \frac{\partial T}{\partial x_{j}}\right) .
\end{aligned}
$$

In these equations, symbols $V_{i}, p, T, x_{i}, t$ represent velocity, pressure, temperature, spatial coordinates and time respectively. The symbols $v$ and $\mathrm{Pr}$ represent kinematic viscosity and the Prandtl number of the fluid medium. Both these quantities are assumed to be constant.
We derive the governing equations of a partiallyaveraged field by subjecting equation set (1) to the so-called partial-averaging filter. This filter is \langle\rangle assumed to be constant preserving and commutative with spatial and temporal differentiation (Girimaji 2006). Subjecting equation set (1) to this filter results into the governing equations of the filtered counterparts of velocity, pressure and temperature ( $\left\langle V_{i}\right\rangle,\langle p\rangle$ and $\langle T\rangle$ respectively):

$$
\begin{gathered}
\frac{\partial\left\langle V_{i}\right\rangle}{\partial x_{i}}=0 ; \quad \frac{\partial\left\langle V_{i}\right\rangle}{\partial t}+\left\langle V_{j}\right\rangle \frac{\partial\left\langle V_{i}\right\rangle}{\partial x_{j}}=-\frac{1}{\rho} \frac{\partial\langle p\rangle}{\partial x_{i}} \\
+\frac{\partial}{\partial x_{j}}\left(v \frac{\partial\left\langle V_{i}\right\rangle}{\partial x_{j}}\right)-\frac{\partial \tau\left(V_{i}, V_{j}\right)}{\partial x_{j}} ; \\
\frac{\partial\langle T\rangle}{\partial t}+\left\langle V_{i}\right\rangle \frac{\partial\langle T\rangle}{\partial x_{i}}=\frac{\partial}{\partial x_{i}}\left(\frac{v}{\operatorname{Pr}} \frac{\partial\langle T\rangle}{\partial x_{i}}\right)-\frac{\partial \tau\left(V_{i}, T\right)}{\partial x_{i}} .
\end{gathered}
$$

The new symbols $\tau\left(\mathrm{V}_{\mathrm{i}}, \mathrm{V}_{\mathrm{j}}\right)$ and $\tau\left(\mathrm{V}_{\mathrm{i}}, \mathrm{T}\right)$ appearing in equation set (2) are called the generalized central moments in accordance with the general filtering representation of the Navier-Stokes equations proposed by Germano (1992):

$$
\begin{aligned}
& \tau\left(V_{i}, V_{j}\right)=\left\langle V_{i} V_{j}\right\rangle-\left\langle V_{i}\right\rangle\left\langle V_{j}\right\rangle ; \\
& \tau\left(V_{j}, T\right)=\left\langle V_{j} T\right\rangle-\left\langle V_{j}\right\rangle\langle T\rangle .
\end{aligned}
$$

The quantity $\tau\left(\mathrm{V}_{\mathrm{i}}, \mathrm{V}_{\mathrm{j}}\right)$ represents the turbulent stress arising because of the filtering operation of the advection process incumbent in the instantaneous momentum equation. Similarly, the quantity $\tau\left(\mathrm{V}_{\mathrm{i}}, \mathrm{T}\right.$ ) is the turbulent thermal flux process arising because of the filtering operation performed on the non-linear advection process incumbent in the temperature equation. Clearly, both $\tau\left(\mathrm{V}_{\mathrm{i}}, \mathrm{V}_{\mathrm{j}}\right)$ and $\tau\left(\mathrm{V}_{\mathrm{i}}, \mathrm{T}\right)$ are unclosed terms in the PANS filtered equation set (2), and thus appropriate turbulence closure models are required to make any computation feasible.

\subsection{Conventional Closure of PANS using Linear Eddy Viscosity Model}

In his proposal of PANS equations and closures, Girimaji (2006) proposed a linear eddy viscosity closure (Boussinesq 1877) for the generalized central moment of the momentum equation (here we present a brief review, the reader is referred to Girimaji (2006) for further details):

$$
\tau\left(V_{i}, V_{j}\right)=\frac{2}{3} k_{u} \delta_{i j}-2 v_{u}\left\langle S_{i j}\right\rangle ;
$$

In this equation, $\left\langle S_{i j}\right\rangle$ represents the filtered strain-rate tensor $\left\langle S_{i j}\right\rangle=\frac{1}{2}\left(\frac{\partial\left(V_{j}\right)}{\partial x_{i}}+\frac{\partial\left(V_{i}\right)}{\partial x_{j}}\right) ; \mathrm{k}_{\mathrm{u}}$ is the unresolved turbulent kinetic energy: $\mathrm{k}_{\mathrm{u}}=\frac{1}{2} \tau$ $\left(\mathrm{V}_{\mathrm{i}}, \mathrm{V}_{\mathrm{i}}\right)$. The symbol $v_{\mathrm{u}}$ is the unresolved eddy viscosity. In line with the nomenclature adopted by 
researchers in the RANS paradigm (Argyropoulos and Markatos 2015; Hellsten and Wallin 2009), equation (4) can be called the linear eddy viscosity model for the unclosed stresses, because the anisotropic part of the closed stress tensor is assumed to be have a linear dependence on the resolved strain-rate. Girimaji (2006) derived the PANS version of the standard k- $\varepsilon$ model of Launder and Spalding (1974) and modeled $v_{\mathrm{u}}$ as:

$v_{u}=C_{\mu} \frac{k_{u}^{2}}{\varepsilon_{u}}, \quad$ where $\varepsilon_{u}=v_{u}\left(\frac{\partial V_{i}}{\partial x_{k}}, \frac{\partial V_{i}}{\partial x_{k}}\right)$

The symbol $\tau\left(\frac{\partial V_{i}}{\partial x_{k}}, \frac{\partial V_{i}}{\partial x_{k}}\right)$ is the generalized central moment of : $\frac{\partial V_{i}}{\partial x_{k}}: \tau\left(\frac{\partial V_{i}}{\partial x_{k}}, \frac{\partial V_{i}}{\partial x_{k}}\right)=\left\langle\frac{\partial V_{i}}{\partial x_{k}} \frac{\partial V_{i}}{\partial x_{k}}\right\rangle-\left\langle\frac{\partial V_{i}}{\partial x_{k}}\right\rangle\left\langle\frac{\partial V_{i}}{\partial x_{k}}\right\rangle$ Further, Girimaji (2006) achieved closure for $\mathrm{k}_{\mathrm{u}}$ and $\varepsilon_{u}$ by including the modelled equations of these quantities $\mathrm{k}_{\mathrm{u}}$ and $\varepsilon_{\mathrm{u}}$.

$$
\begin{aligned}
\frac{\partial k_{u}}{\partial t}+\left\langle V_{j}\right\rangle \frac{\partial k_{u}}{\partial x_{j}} & =P_{u}-\varepsilon_{u}+\frac{\partial}{\partial x_{j}}\left(\left(\frac{v_{u}}{\sigma_{k u}}+v\right) \frac{\partial k_{u}}{\partial x_{j}}\right) ; \\
\frac{\partial \varepsilon_{u}}{\partial t}+\left\langle V_{j}\right\rangle \frac{\partial \varepsilon_{u}}{\partial x_{j}} & =\left(C_{e 1} \frac{P_{u} \varepsilon_{u}}{k_{u}}-C_{e 2}^{*} \frac{\varepsilon_{u}^{2}}{k_{u}}\right) \\
& +\frac{\partial}{\partial x_{j}}\left(\left(\frac{v_{u}}{\sigma_{\varepsilon u}}+v\right) \frac{\partial \varepsilon_{u}}{\partial x_{j}}\right)
\end{aligned}
$$

Here $\mathrm{P}_{\mathrm{u}}$ is the unresolved production term $\left(=-\tau\left(V_{i} V_{j}\right) \frac{\partial\left\langle V_{i}\right\rangle}{\partial x_{j}}\right)$. The quantities $f_{k}$ and $f_{\varepsilon}$ appearing in these equations are the implicit filter parameter of the PANS methodology. These parameters are defined as: $f_{k}=\left(k_{u} / k\right) ; f_{\varepsilon}=\left(\varepsilon_{u} / \varepsilon\right)$; where $k$ and $\varepsilon$ represent the turbulent kinetic energy and its dissipation rate (in RANS sense). Various coefficients appearing in equation set (6) are:

$$
\text { ; } \quad C_{e 2}^{*}=C_{e 1}+\left(f_{k} / f_{\varepsilon}\right)\left(C_{e 2}-C_{e 1}\right)
$$

$; \sigma_{k u}=\left(\sigma_{k} f_{k}^{2} / f_{\varepsilon}\right) ; \sigma_{\varepsilon u}=\left(\sigma_{\varepsilon} f_{k}^{2} / f_{\varepsilon}\right)$

$\mathrm{C}_{\mathrm{e} 1}=1.44, \mathrm{C}_{\mathrm{e} 2}=1.92, \sigma_{\varepsilon}=1.3, \sigma_{\mathrm{k}}=1$.

For purely hydrodynamic problems, wherein it is not required to solve the temperature equation, equation set $(2,4,6)$ form a closed set of equations. The filter parameters $f_{k}$ and $f_{\varepsilon}$ can vary between 0 and 1 , allowing the user to implicitly choose the resolved-to-unresolved cutoff length scale. Setting $f_{k}=0$ and $f_{\varepsilon}=0$ converts the formulation into instantaneous Navier-Stokes equation set. On the other extreme, setting $f_{k}=1$ and $f_{\varepsilon}=1$ converts the formulation to the Reynolds-averaged NavierStokes (RANS) equation set. Setting $0<f k<1,0<$ $f_{\varepsilon}<1$ results into the so-called partially averaged Navier-Stokes equation description (Girimaji 2006), wherein the filter cutoff is implicitly lying in the inertial range of the turbulence kinetic energy spectrum. The reader is referred to Girimaji (2006) for further details about the filter parameters of PANS.

\subsection{Conventional Closure of the Temperature Equation}

Girimaji (2006) in his proposal did not include the temperature equation. However, recently Ranjan and Dewan (2015) extended the conventional PANS closure to heat transfer problems. The authors made a straight-forward modification of the conventional linear gradient diffusion hypothesis of the RANS paradigm:; here, $\left(\overline{V_{i}^{\prime} T^{\prime}}\right)=-\frac{v_{T}}{\operatorname{Pr}_{T}} \frac{\partial T}{\partial x_{i}}$ an overbar implies a mean value and a prime implies fluctuation (in RANS sense). To model the unclosed thermal flux process in the PANS-filtered temperature equation as:

$$
\tau\left(V_{i}, T\right)=-\frac{v_{u}}{\operatorname{Pr}_{T}} \frac{\partial\langle T\rangle}{\partial x_{i}}
$$

where $\operatorname{Pr}_{T}$ is the turbulent Prandtl number. The quantity $\left(v_{u} / \operatorname{Pr}_{T}\right)$ can be called unresolved thermal eddy diffusivity. With the equation set (2, 4, 6 and 7 ), the conventional PANS closure is achieved for simulating incompressible flows with heat transfer. We refer to this closed equation set $(2,4,6$ and 7) as the linear eddy viscosity model+ PANS (or LEVM+PANS) methodology for simulating incompressible flows with heat transfer.

\subsection{Closure using Non-Linear Turbulent Stresses}

In this subsection, we present an alternate closure of the PANS filtered equations invoking the concept of the so called non-linear turbulent stresses. The idea of non-linear turbulent stresses was originally proposed in context of RANS closure as an attempt to address the short-comings of the linear eddy viscosity model of Boussinesq (1877). In the formulation proposed by Lumley (1970) and later utilized by Shih et al. (1993), the Reynolds stress tensor $\left(\overline{V_{i}^{\prime} V_{j}^{\prime}}\right)$ is expressed as a function of the mean strain-rate as well as higher order tensor products of the mean strain-rate and the mean rotation-rate tensors as well. Such a modeling paradigm not only allows the turbulent stresses to have potentially more accurate dependence on the mean strain-rate tensor but also on the mean rotation-rate tensor, the effect of which is entirely ignored in the Boussinesq's linear eddy viscosity model. These higher order terms are collectively called the non-linear turbulent stresses. Including higher order terms in the constitutive equation of the turbulent stress tensor allows the turbulent stress to break away from the over-simplifying assumption of the linear eddy viscosity model wherein the anisotropic part of the turbulent stress tensor is unconditionally forced to align with the local mean strain-rate tensor. Experimental evidence does show that this assumption of perfect 
alignment between the turbulent stress tensor and the mean strain-rate tensor indeed breaks down over substantial regions in a separated flows past bluff bodies (Chesnakas et al. 1997). Recently, Yangaz et al. (2019) has employed non-linear eddy viscosity model in conjugation with RANS to simulate and evaluate performance of various burners designs and choice of fuels.

Over the last three decades, several non-linear models have been proposed and tested for closure of RANS momentum equations (Speziale 1987; Rubinstein and Barton 1990; Craft et al. 1996; Apsley et al. 1997; Ramesh et al. 2006; Colombo et al. 2008; Gatski and Jongen 2000; Hellsten and Wallin 2009; Shih et al. 1993). Among these, Shih's model (Shih et al. 1993) has been employed in a variety of flow fields showing significant improvements. In this work we focus on the Shih's non-linear model which employs a combination of linear and quadratic non-linear stress in conjugation with the k- $\varepsilon$ model. Since the original version of this model is meant for RANS closure, our next task in this section is to appropriately extract the PANS version of this closure.

Using the quadratic constitutive relation proposed by Shih et al. (1993), in place of Boussinesq's approximation for Reynolds stress, we model $\tau\left(\mathrm{V}_{\mathrm{i}}, \mathrm{V}_{\mathrm{j}}\right)$ as:

$$
\begin{aligned}
\tau\left(V_{i}, V_{j}\right)= & \frac{2}{3} k_{u} \delta_{i j}-v_{u}\left(\left\langle V_{i}\right\rangle_{j}+\left\langle V_{j}\right\rangle_{i}\right) \\
& +\frac{C_{r 1}}{A_{2}+\eta^{3}} \frac{k_{u}^{2}}{\varepsilon_{u}^{2}}\left(\left\langle V_{i}\right\rangle_{k}\left\langle V_{k}\right\rangle_{j}\right. \\
& \left.+\left\langle V_{j}\right\rangle_{k}\left\langle V_{k}\right\rangle_{i}-\frac{2}{3} \Pi \delta_{i j}\right) \\
& +\frac{C_{r 2}}{A_{2}+\eta^{3}} \frac{k_{u}^{2}}{\varepsilon_{u}^{2}}\left(\left\langle V_{k}\right\rangle_{j}\left\langle V_{k}\right\rangle_{j}-\frac{1}{3} \tilde{\Pi} \delta_{i j}\right) \\
& +\frac{C_{r 3}}{A_{2}+\eta^{3}} \frac{k_{u}^{2}}{\varepsilon_{u}^{2}}\left(\left\langle V_{k}\right\rangle_{i}\left\langle V_{k}\right\rangle_{j}-\frac{1}{3} \tilde{\Pi} \delta_{i j}\right)
\end{aligned}
$$

$$
\begin{aligned}
& \text { where } \mathrm{v}_{u}=C_{\mu} \frac{k_{u}^{2}}{\varepsilon_{u}} ; C_{\mu}=\frac{2 / 3}{A_{1}+\eta+\alpha \xi} ; \eta=\frac{k_{u} S}{\varepsilon_{u}} ; \\
& S=\left(2\left\langle S_{i j}\right\rangle\left\langle S_{i j}\right\rangle\right)^{1 / 2} ;\left\langle S_{i j}\right\rangle=\frac{\left(\left\langle V_{i}\right\rangle_{j}+\left\langle V_{j}\right\rangle_{i}\right)}{2} ; \xi=\frac{k_{u} \Omega}{\varepsilon_{u}} ; \\
& \Omega=\left(2\left\langle\Omega_{i j}\right\rangle\left\langle\Omega_{i j}\right\rangle\right)^{1 / 2} ;\left\langle\Omega_{i j}\right\rangle=\frac{\left(\left\langle V_{i}\right\rangle_{j}+\left\langle V_{j}\right\rangle_{i}\right)}{2} ; \\
& \left\langle V_{i}\right\rangle_{j}=\frac{\partial\left\langle V_{i}\right\rangle}{\partial x_{j}} ; \Pi=\left\langle V_{i}\right\rangle_{j}\left\langle V_{j}\right\rangle_{i} ; \tilde{\Pi}=\left\langle V_{i}\right\rangle_{j}\left\langle V_{j}\right\rangle_{i} .
\end{aligned}
$$

The additional coefficients used in equations set (8) have the values: $C_{\mathrm{r} 1}=-4, \mathrm{C}_{\mathrm{r} 2}=13, \mathrm{C}_{\mathrm{r} 3}=-2, \mathrm{~A}_{1}=$ $1.25, \mathrm{~A}_{2}=1000$ and $\alpha=0.09$. Note that equation (8) when combined with the evolution equations of $\mathrm{k}_{\mathrm{u}}$ and $\varepsilon_{\mathrm{u}}$ (equation set 6) and the filtered continuity and the filtered momentum equations set (2) form a closed set of equations for the the hydrodynamic flow field. In equation (8) the third, fourth and the fifth group of terms are collectively called as the non-linear turbulent stress. The non-linear stress depends on the quadratic tensorial products of the resolved strain-rate and the rotation-rate tensors. The non-linear stress is added over and above to the so-called linear part of the stress (second term in equation 8). Due to the inclusion of the non-linear stress in equation (8), Argyropoulos and Markatos (2015) in their review article recommend calling such a closure as a non-linear eddy viscosity model (NLEVM). In contrast, we would describe the conventional Boussinesq's closure (equation 4) as a linear eddy viscosity model (LEVM).

\section{2d. An NLEVM-Based Closure of the Temperature Equation}

The conventional closure of the filtered temperature equation as employed by Ranjan and Dewan (2015) in their heat transfer study (using linear eddy viscosity closure for both momentum and temperature equations) is:

$$
\tau\left(V_{i}, T\right)=-\frac{v_{u}}{\operatorname{Pr}_{T}} \frac{\partial\langle T\rangle}{\partial x_{i}}
$$

However, we suggest that in the light of the fact that the filtered momentum equation has now been closed using a superior representation of turbulent stresses equation (8), equation (9) may not be an optimal model in the given non-linear framework. Thus, with the intention to directly sensitize the closure of turbulent thermal flux to the presence of non-linear stresses in the formulation, we propose to modify equation (9).

Equation (9) can be expressed as:

$$
\tau\left(V_{i}, T\right)=-\frac{v_{u}}{\operatorname{Pr}_{T}} \frac{\partial\langle T\rangle}{\partial x_{i}}=-\frac{v}{v_{u}}\left[\frac{v}{\operatorname{Pr}_{T}} \frac{\partial\langle T\rangle}{\partial x_{i}}\right]
$$

In the last term, the factor $v_{\mathrm{u}} / v$ has been extracted out to underline the modelling philosophy: the factor $v_{\mathrm{u}} / v$ is expected to capture the eddy-enhanced diffusive transport of thermal energy (compared to the molecular transport). Note that this factor of $v_{\mathrm{u}} / v$ is also the ratio of the magnitude of the anisotropic portions of the turbulent stress to the filtered viscous stress when a linear eddy viscosity model like equation (4) is used:

$$
\frac{\left\|\tau^{\text {anisotropic }}\left(V_{i}, V_{j}\right)\right\|}{\left\|\tau_{p q}^{\text {viscous }}\right\|}=\frac{\left\|2 v_{u}\left\langle S_{i j}\right\rangle\right\|}{\left\|2 v_{u}\left\langle S_{p q}\right\rangle\right\|}=\frac{v_{u}}{v}
$$

where $\left\|\mathrm{A}_{\mathrm{ij}}\right\|=\sqrt{\mathrm{A}_{\mathrm{ij}} \mathrm{A}_{\mathrm{ij}}}$ (magnitude of a secondorder tensor, $A_{i, j}$ ). Clearly, when an LEVM model is used to close the filtered momentum equation, $v_{\mathrm{u}} / v$ also represents the ratio of the magnitude of turbulent stresses to that of the filtered viscous stresses. Note that it is the ratio of the magnitudes of the turbulent to filtered viscous stresses which actually model the diffusive effect of turbulent eddies in the filtered momentum equation.

However, in contrast, when an NLEVM (like equation 8) is used to close the filtered momentum equation, the ratio of the magnitude of the turbulent to filtered viscous stresses cannot be written simply as the $v_{\mathrm{u}} / v$ : 


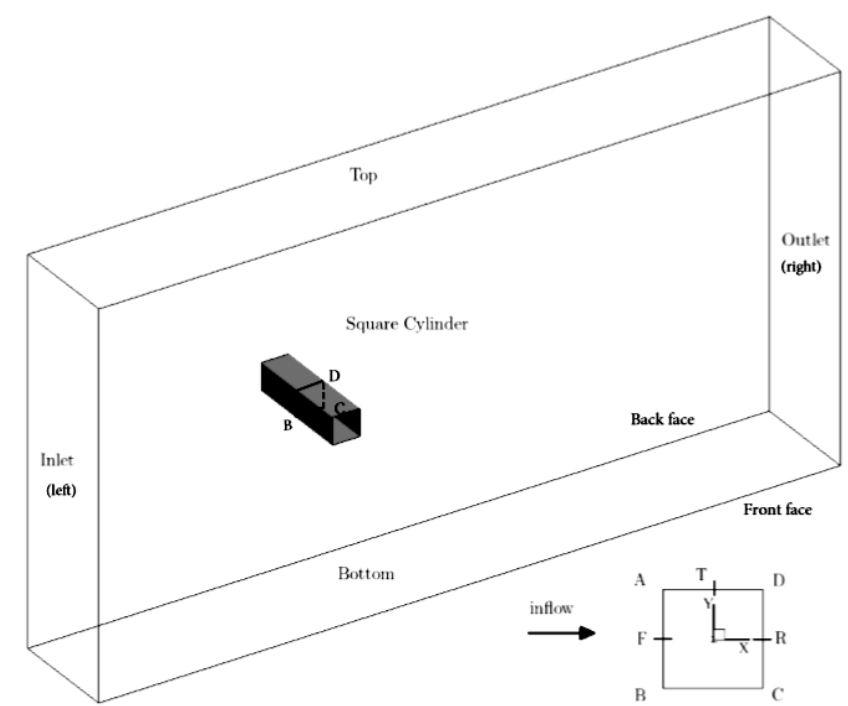

Fig. 1. Computational domain (the origin of the working coordinate system lies at the mid-span location of the cylinder).

$$
\frac{\left\|\tau^{\text {anisotropic }}\left(V_{i}, V_{j}\right)\right\|}{\left\|\tau_{p q}^{\text {viscous }}\right\|}=\frac{\left\|2 v_{u}\left\langle S_{i j}\right\rangle+N_{i j}\right\|}{\left\|2 v_{u}\left\langle S_{p q}\right\rangle\right\|} \neq \frac{v_{u}}{v} \text { (12) }
$$

where $N_{i j}$ represents the $(i-j)^{\text {th }}$ component of the non-linear part of the turbulent stress tensor presented in equation (8):

$$
\begin{aligned}
N_{i j} & =\frac{C_{r 1}}{A_{2}+\eta^{3}} \frac{k_{u}^{3}}{\varepsilon_{u}^{2}}\left(\left\langle V_{i}\right\rangle_{k}\left\langle V_{k}\right\rangle_{j}\right. \\
& \left.+\left\langle V_{j}\right\rangle_{k}\left\langle V_{k}\right\rangle_{i}-\frac{2}{3} \Pi \delta_{i j}\right) \\
& +\frac{C_{r 2}}{A_{2}+\eta^{3}} \frac{k_{u}^{2}}{\varepsilon_{u}^{2}}\left(\left\langle V_{i}\right\rangle_{k}\left\langle V_{j}\right\rangle_{k}-\frac{1}{3} \tilde{\Pi} \delta_{i j}\right) \\
& +\frac{C_{r 3}}{A_{2}+\eta^{3}} \frac{k_{u}^{3}}{\varepsilon_{u}^{2}}\left(\left\langle V_{k}\right\rangle_{i}\left\langle V_{k}\right\rangle_{j}-\frac{1}{3} \tilde{\Pi} \delta_{i j}\right)
\end{aligned}
$$

Equation (12) suggests that in the presence of an NLEVM model, $v_{\mathrm{u}} / v$ is no more an optimal measure of the diffusive effects of turbulent stresses in the momentum equation. Thus, in the presence of an NLEVM model, we propose that the amplification of eddy-diffusivity in the filtered temperature equation must also be measured not in terms of $v_{\mathrm{u}} / v$ , but in terms of the actual stress ratio (equation 12). Accordingly, we propose the following model for the unclosed thermal flux as:

$$
-\tau\left(V_{i}, T\right)=\Gamma \frac{v}{\operatorname{Pr}_{T}} \frac{\partial\langle T\rangle}{\partial x_{i}}
$$

where

$$
\Gamma=\frac{\left\|\tau^{\text {anisotropic }}\left(V_{i}, V_{j}\right)\right\|}{\left\|\tau_{p q}^{\text {viscous }}\right\|}=\frac{\left\|2 v_{u}\left\langle S_{i j}\right\rangle+N_{i j}\right\|}{\left\|2 v\left\langle S_{p q}\right\rangle\right\|} . \mathrm{We}
$$

refer to ratio $\Gamma$ as the stress ratio (SR). This quantity, in general, will depend on both space and time and allows the thermal flux model to be sensitive to the presence of non-linear stresses in the flow field. Due to this dependence of the model on the non-linear stresses, and in turn on the non-linear tensorial products of the resolved strain-rate and rotation-rate tensors as well, equations (8) and (14) can be described as an implicitly non-linear constitutive equation. The authors' intent is not to claim that this model (14) is necessarily the "best possible model" of thermal flux. The authors acknowledge that possibly a more advanced PANS closure can be derived using an anisotropy-based approach - like that of Daly and Harlow (1970), Abe et al. (1994), Abe et al. (1995)- or even an evolution equation based approach (like that of Wikström et al. (2000)). The intent of this modified proposal (14) is merely to propose and explore a more appropriate zero-equation model (in comparison with equation (9)) for the unclosed thermal flux when an NLEVM model is being used in the filtered momentum equation.

The equation set of $(2,6,8)$ along with the new model (14) is now closed, and we refer to this set as the NLEVM+PANS+SR methodology. In contrast, we refer to the closed set of equations comprising of $(2,6,8)$ along with the linear version of the thermal flux model (9) as the NLEVM+PANS methodology.

\section{COMPUTATIONAL SET-UP}

All our evaluations are performed for the flow past a square cylinder at Reynolds number $(\mathrm{Re})=21,400$. The computational domain used for these simulations has dimensions: 27D stream-wise, 14D cross-stream-wise and 4D span-wise (where D is the length of the base side of the square cylinder). The origin of our coordinate system is located at the centre of the square cylinder (at location 9D downstream from the inlet). A schematic of the computational domain is presented in the Fig. 1. 

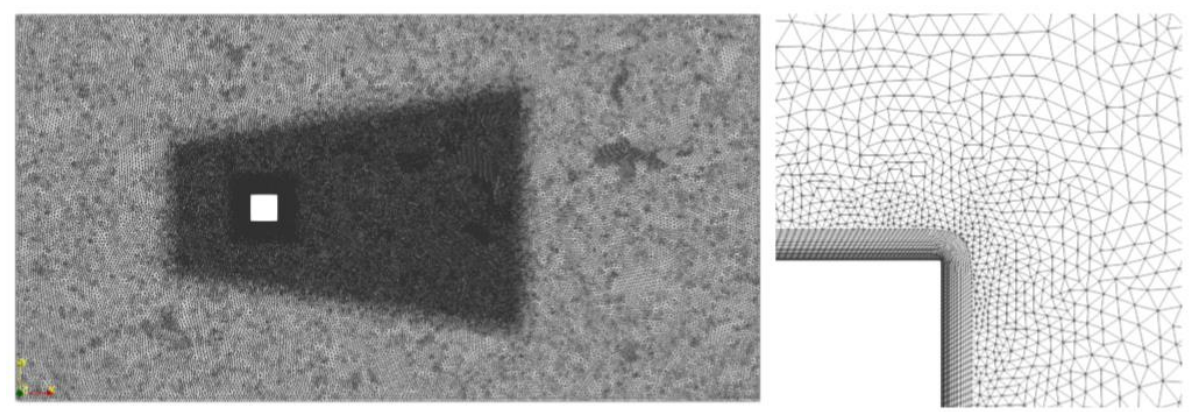

Fig. 2. (a) Side view of mid z-plane slice of grid (left), (b) Near surface mesh (zoomed view) on the square cylinder (right).

This choice of domain is in line with the computational studies (Nakayama and Vengadesan 2002; Song and Park 2009; Jeong and Girimaji 2010; Ranjan and Dewan 2015; Basara and Girimaji 2018). The equations (2, 4 or 8,6 and 7 or 14$)$ are solved throughout the computational domain.

At inlet (left face of domain), the boundary conditions are: velocity specified as $1 \mathrm{~m} / \mathrm{s}$ along $\mathrm{x}$ direction, zero pressure gradient, temperature as $300 \mathrm{~K}$, turbulent kinetic energy $(\mathrm{k})$ as $6.0 \mathrm{e}-4 \mathrm{~m}^{2} / \mathrm{s}^{2}$ and turbulent dissipation rate $(\varepsilon)$ as $6.9 \mathrm{e}-5 \mathrm{~m}^{2} / \mathrm{s}^{3}$. Top and bottom faces have slip boundary condition for velocity, zero pressure gradient, and temperature, $\mathrm{k}$ and $\varepsilon$ have the same values as at the inlet. The two lateral walls (front and back) are given cyclic boundary conditions. At outlet (right face of domain) the boundary conditions are: zero value of gauge pressure, and zero gradient for velocity, temperature, $\mathrm{k}$ and $\varepsilon$. The four surfaces of the square cylinder have no-slip boundary condition for velocity, zero pressure gradient, and temperature as $330 \mathrm{~K}$.

A wall-resolved unstructured mesh with uniform extrusion in the span-wise direction (prepared using ANSYS ICEM software), is employed for our study. The near-wall regions are provided with inflation layers. The first cell height is chosen to be small enough to ensure that it is in the viscous layer with $\mathrm{y}^{+}$value less than unity (and $\mathrm{x}^{+}$value less than 20). A conformal interface between the inflation layers and the tetrahedral grid cells is ensured. As we move away from the cylinder walls, a growth ratio of 1.05 is provided. The planar grid consists of 0.15 million cells. This planar grid is provided an extrusion in the z-direction with 32 nodes to obtain a three-dimensional grid $\left(\mathrm{z}^{+}\right.$value less than 200$)$. The resolution in the $\mathrm{z}$-direction is comparable to that used by previous works employing PANS simulations (Jeong and Girimaji 2010; Ranjan and Dewan 2015; Basara and Girimaji 2018) for the flow past a square cylinder at the same Reynolds number (as the one used in our work). The total cell count of this grid is around 5 million. This grid is primarily employed for our simulations. A representative view of this grid is presented in Fig. 2.

All computations are performed using the OpenFOAM computational package. Appropriate edits and additions in the solver scripts have been made to suitably incorporate the LEVM+PANS, NLEVM+PANS and NLEVM+PANS+SR methodologies. In all our simulations, near wall treatment is achieved using the two-layer treatment proposed by Chen and Patel (1988). The discretization schemes employed in our simulations along with other relevant solver settings are mentioned in Table 1 .

Table 1 Details of schemes and solver

\begin{tabular}{|c|c|}
\hline & Schemes \\
\hline Temporal & $2^{\text {nd }}$ Order Implicit \\
\hline Gradient (default) & $2^{\text {nd }}$ Order Linear \\
\hline Gradient (pressure) & Least-squares \\
\hline Divergence & Bounded $2^{\text {nd }}$ order upwind \\
\hline Laplacian & $2^{\text {nd }}$ Order Linear \\
\hline Interpolation & Linear-corrected \\
\hline Surface-normal Gradient & Linear \\
\hline Residual Tolerances & $1 \mathrm{e}-06$ \\
\hline
\end{tabular}

\begin{tabular}{|c|c|}
\hline $\begin{array}{c}\text { Pressure-Velocity } \\
\text { coupling }\end{array}$ & $\begin{array}{c}\text { PISO (Pressure Implicit with } \\
\text { Splitting of Operators) algorithm }\end{array}$ \\
\hline Variable & Solver \\
\hline Pressure & $\begin{array}{c}\text { Geometric agglomerated } \\
\text { Algebraic MultiGrid (GAMG) }\end{array}$ \\
\hline Smoother & $\begin{array}{c}\text { Diagonal based Incomplete Cholesky } \\
\text { Gauss Siedel (DICGaussSiedel) }\end{array}$ \\
\hline $\begin{array}{c}\text { Velocity, } k, \varepsilon \\
\text { and temperature }\end{array}$ & $\begin{array}{c}\text { Pre-conditioned Bi-Conjugate } \\
\text { Gradient Stabilized (PBiCGStab) }\end{array}$ \\
\hline Pre-conditioner & $\begin{array}{c}\text { Diagonal based Incomplete LU } \\
\text { (DILU) }\end{array}$ \\
\hline
\end{tabular}

Evaluation of LEVM+PANS, NLEVM+PANS and NLEVM+PANS+SR methodologies are performed employing unsteady three-dimensional simulations. In Table 2 we include a brief description indicating value of parameter $\left(f_{k}\right)$ and the methodologies used in these simulations. All these simulations are performed using the three dimensional grid described earlier in section 3 (Fig. 2). For all these simulations, the other filter parameter, $f_{\varepsilon}$ is set to unity as the Reynolds number is high enough to justify almost all dissipation to be concen trated in the smallest (modelled) scales of motion (Girimaji 2006; Jeong and Girimaji 2010). Note that simulations $\mathrm{A}$ and $\mathrm{D}$ which have $f_{k}=1$ are actually unsteady RANS simulations. Merely for the sake of having uniformity in notation, we refer to these 


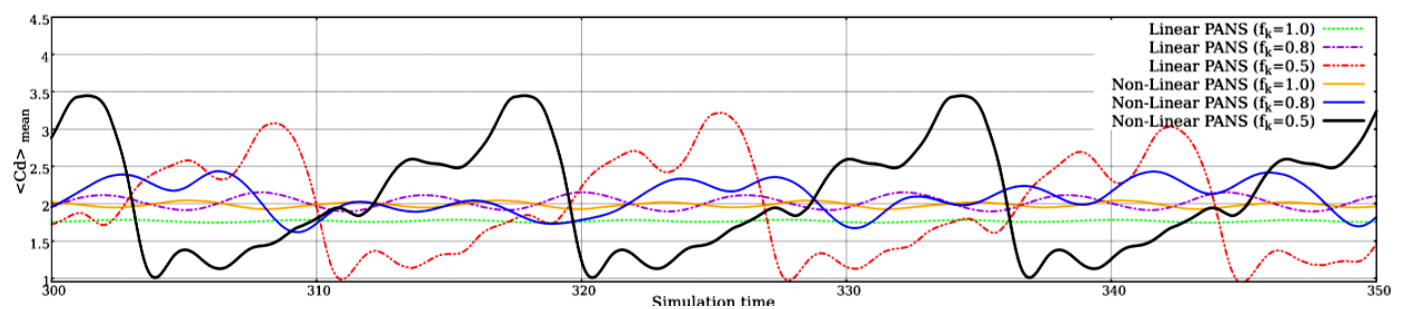

Fig. 3. Temporal variation in $\left\langle\mathrm{C}_{\mathrm{d}}\right\rangle_{\text {mean }}$ obtained from various simulations.

simulations as LEVM+PANS with $f_{k}=1$ and NLEVM+PANS with $f_{k}=1$. On the other hand, simulations $\mathrm{B}, \mathrm{C}, \mathrm{E}, \mathrm{F}$ and $\mathrm{G}$ are PANS simulations, because $f_{k}<1$. Results of simulation cases A-F are used to evaluate the performance of the LEVM+PANS and NLEVM+PANS methodologies. Results of simulations $\mathrm{F}$ and $\mathrm{G}$ are compared to identify the improvements in the ability to capture heat transfer characteristics with the SR-based model of the thermal flux process. Even though the PANS methodology allows $f_{k}$ to be varied between 1 and 0 , our study employs only three values of $f_{k}(1,0.8$ and 0.5$)$. Since the intent of this work is not necessarily to report the best predictions by the PANS methodology, but rather to demonstrate the similarity and differences in the inherent capabilities of the linear and the nonlinear PANS, we deem our PANS simulations up to even $f_{k}=0.5$ to be adequately serving the objectives of this study.

Table 2 Simulation cases

\begin{tabular}{|c|c|c|}
\hline Case & Simulation & $f_{k}$ \\
\hline $\mathrm{A}$ & LEVM+PANS & 1 \\
\hline $\mathrm{B}$ & LEVM+PANS & 0.8 \\
\hline $\mathrm{C}$ & LEVM+PANS & 0.5 \\
\hline $\mathrm{D}$ & NLEVM+PANS & 1 \\
\hline $\mathrm{E}$ & NLEVM+PANS & 0.8 \\
\hline $\mathrm{F}$ & NLEVM+PANS & 0.5 \\
\hline $\mathrm{G}$ & NLEVM+PANS+SR & 0.5 \\
\hline
\end{tabular}

\section{QUANTITIES OF INTEREST AND TIME-STEP/GRID SENSITIVITY}

Each simulation starts with a uniformly initialized flow field which is then allowed to develop over ten flow-through times $\left(\mathrm{tV}_{0} / 27 \mathrm{D}\right.$, where $\mathrm{V} 0$ is the farupstream velocity). By this time, the flow attains a state after which time-averaging of various quantities of interest begins. Subsequently, the simulations are continued further for next ten flowthrough times. All mean quantities reported in this work are time-averaged values obtained over the latter ten flow-through times only. In Fig. 3, we present temporal variations in $\mathrm{C}_{\mathrm{d}}$ obtained from Simulation A-F. Plots are shown from 300 to 350 flow simulation time. Quantitative evaluations are performed using these time-averaged values of quantities of interest. Some qualitative comparisons and physical explanations are sought using instantaneous flow fields as well.
The mean quantities of our interest are: (i) mean Nusselt number on the cylinder surface $\left(\langle N u\rangle_{\text {mean }}\right)$, (ii) mean coefficient of drag $\left(\left\langle C_{D}\right\rangle_{\text {mean }}\right)$, (iii) mean coefficient of pressure $\left(\left\langle C_{p}\right\rangle_{\text {mean }}\right)$ along the cylinder surface, (iv) recirculation length behind the cylinder $\left(\mathrm{L}_{\mathrm{r}}\right)$, (b) mean stream-wise velocity profiles, and (vii) second-order turbulence statistics in the flow field $\left(u_{i}^{\prime} u_{i}^{\prime}\right)$.

$$
\begin{aligned}
\langle N u\rangle_{\text {mean }}= & \frac{\left\langle\dot{q}_{\text {wall }} \mid\right\rangle_{\text {mean }} D}{K\left(T_{\text {wall }}-T_{0}\right)}=\frac{D}{K\left(T_{\text {wall }}-T_{0}\right)}\left|\frac{\partial\langle\langle T\rangle\rangle_{\text {mean }}}{\partial n}\right| \\
\left\langle C_{D}\right\rangle_{\text {mean }}= & \frac{\left\langle F_{x}\right\rangle_{\text {mean }}}{\frac{1}{2} \rho V_{0}^{2} 4 D^{2}} ; \quad\left\langle C_{p}\right\rangle_{\text {mean }}=\frac{\langle\langle p\rangle\rangle_{\text {mean }}-p_{0}}{\frac{1}{2} \rho V_{0}^{2}} ; \\
& u_{i}^{\prime} u_{i}^{\prime}=\left\langle\left(\left\langle V_{i}\right\rangle-\left\langle\left\langle V_{i}\right\rangle\right\rangle_{\text {mean }}\right)^{2}\right\rangle_{\text {mean }}
\end{aligned}
$$

Note that the symbol \langle\rangle$_{\text {mean }}$ means time-averaged value (henceforth referred to as "mean values") of the quan-tity in context. The symbols $n, K, \dot{\mathrm{q}}_{\text {wall }}$, $F_{x}, p_{0}, V_{0}$ and $T_{0}$ represents wall normal distance, thermal conductivity, wall heat flux, net force on the cylinder in x-direction, pressure, velocity and temperature at far upstream locations respectively. In all our simulations, $T_{\text {wall }}=330 \mathrm{~K}$ and $\mathrm{T}_{0}=300 \mathrm{~K}$. The turbulent Prandtl number $\left(\operatorname{Pr}_{\mathrm{T}}\right)$ is set to be unity in all simulations as done in previous computational works (Ranjan and Dewan 2015). All computations are performed at $\operatorname{Re}_{\mathrm{D}}=\frac{\mathrm{V}_{0} \mathrm{D}}{v}=21,400$.

The non-dimensional time step $\left(\Delta \mathrm{t} \mathrm{V}_{0} / \mathrm{D}\right)$ used in simulations $A-G$ is in $0.7-1.2 \times 10^{-3}$ range, which is similar to the time step range $\left(=1.00-1.67 \times 10^{-3}\right)$ used by Cao and Tamura (2016) in their LES studies of flow past a square cylinder at the same Reynolds number. The choice of time-step range is made accounting for balance between temporal resolution and computational resources from engineering point of view - ensuring the maximum Courant number is less than 2. To further ascertain the adequacy of the time steps employed in our simulations, we have performed NLEVM+PANS simulation case with Courant number of 4 . The < $\mathrm{C}_{\mathrm{D}}>_{\text {mean }}$ value obtained from simulation $\mathrm{G}$ is 2.25 , which is merely $1.27 \%$ different from the $<\mathrm{C}_{D}$ $>_{\text {mean }}=2.20$ value obtained from simulation with Courant number of 4 . To further scrutinize time step sensitivity, we follow the rec dence of Cao and 
Tamura (2016) and examine the $\left\langle\mathrm{C}_{\mathrm{p}}\right\rangle_{\text {mean }}$ variation over the cylinder surface as well in Fig. 4. Evidently, no significant differences are observed when Courant number is changed from 2 to 4 . Thus, based on these results, we deem Courant number = 2 to be adequate for the rest of the study.
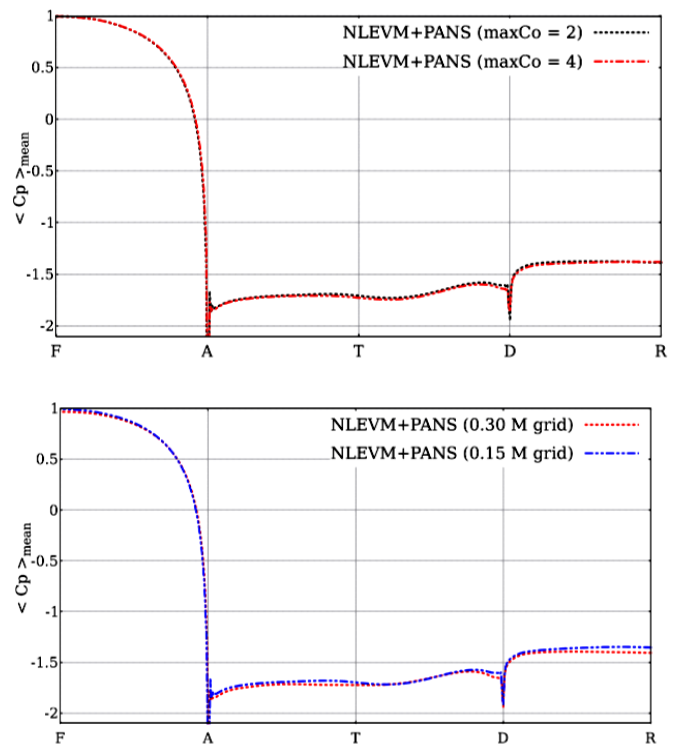

Fig. 4. Locations of points $F$ (mid point of front cylinder face), A, T (mid point of top face), D and $R$ (mid point of rear face) marked on Fig. 1.

The computational grid employed in simulations A$G$ has been generated by uniformly extruding unstructured grid along the span wise $(z)$ direction. The grid in planes parallel to the $x-y$ axes is unstructured. To ascertain the adequacy of our grid for our study, we have performed two identical simulations which differ only in terms of the level of the refinement of the planar grids - one of the same resolution as discussed above [0.15 million (M) cells] and another one with further refinement [with 0.30 million (M) cells]. These simulations are performed with NLEVM+PANS methodology. In Fig. 4 we present the variation of $\left\langle\mathrm{C}_{\mathrm{p}}\right\rangle_{\text {mean }}$ on the cylinder surface in these simulations. Results from the two simulations are sufficiently close.

Further, we have performed the posterori test of PANS on the 0.15 million grid with 32 cells in the lateral direction following the guidelines prescribed by Abdol-Hamid and Girimaji (2004). In the posterori test, the probability density function of the ratio $\mathrm{ku} / \mathrm{k}$ is computed after a PANS simulation has been completed. Data of $k_{u}$ is obtained from our PANS simulation $F$, whereas the data of $k$ is obtained from the corresponding RANS simulation (Simulation D). The input $f_{k}$ value for the PANS simulation is 0.5. In Fig. 5, we present the PDF of $\mathrm{k}_{\mathrm{u}} / \mathrm{k}$. We find that the PDF peaks close to 0.5 , which is the input value of $f_{k}$ for Simulation F. This suggests that our computational grid is adequate for performing a PANS simulation with $f_{k} \geq 0.5$. Based on these results, we deem the planar grid of 0.15 million (M) along with 32 nodes in the span-wise direction to be adequate for the rest of the study. Simulations A-G employ this grid (snapshots of this grid are presented in Fig. 2).

\section{RESULTS AND DISCUSSION}

We begin our discussion with surface-related quantities. In Table 3 , we present $\left\langle\mathrm{C}_{\mathrm{D}}\right\rangle_{\text {mean }}$ values from simulations $A-G$. When $f_{k}$ is reduced, the implicit filter cut-off is supposed to move from largest scales to smaller scales, thus releasing more scales of motion, and purportedly, improving the prediction of mean quantities of a flow field. For LEVM+PANS with $\mathrm{f}_{\mathrm{k}}=1.0$ (simulation $A$ ), the $\left\langle\mathrm{C}_{\mathrm{D}}\right\rangle_{\text {mean }}$ value is 1.76 . As $\mathrm{f}_{\mathrm{k}}$ is reduced to $0.5,\left\langle\mathrm{C}_{\mathrm{D}}\right\rangle_{\text {mean }}$ value predicted by LEVM+PANS approaches 2.07 which is very close to experimental (Lyn et al. 1995) value of 2.11. On the other hand, NLEVM+PANS simulation with $\mathrm{f}_{\mathrm{k}}=1.0$ has the $\left\langle\mathrm{C}_{\mathrm{D}}\right\rangle_{\text {mean }}$ value of 2.02 (which is already matching the prediction of LEVM+PANS at a lower $f_{k}$ value, 0.8). Further, as $f_{k}$ is reduced, NLEVM+PANS $\left(f_{k}=0.5\right)$ shows improvement and predicts value of 2.25 which lies in the range $\left(\left\langle\mathrm{C}_{\mathrm{D}}\right\rangle_{\text {mean }}=2.11\right.$ to $2.30)$, predicted by recent computational works (DNS by Trias et al. (2015)).

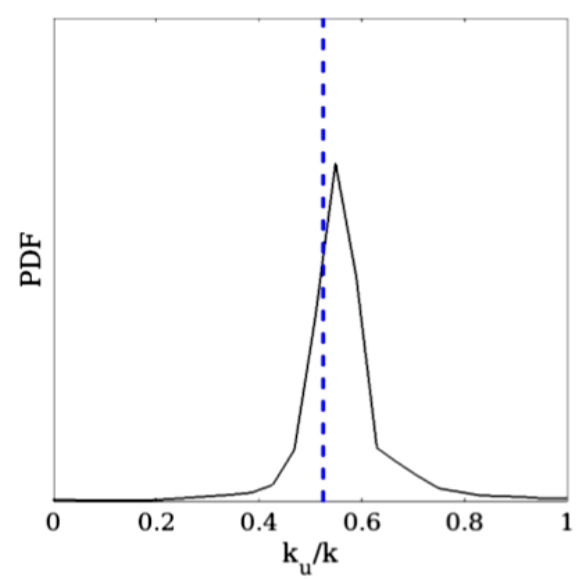

Fig. 5. PDF of $\frac{k_{u}}{k}$ computed using results from Simulations D and F.

In Fig. 6, we plot $\left\langle\mathrm{C}_{\mathrm{D}}\right\rangle_{\text {mean }}$ along segments $\mathrm{AFB}$, BC, CRD and DTA (see Fig. 1). Results from DNS (Trias et al. 2015), wherever available, are used along with experimental results of (Igarashi 1984). We observe that on the front face AFB of the cylinder, performance of all simulations (A-F) are close to the experimental measurements (Igarashi 1984). There is not much difference between the PANS results at $f_{k}=1$ and $f_{k}=0.8$. Hence, results of simulation B and E - both with $f_{k}$ value of $0.8^{-}$are excluded from plots, to avoid cluttering.

On the rear (CRD) side, clearly the performance of LEVM+PANS with $f_{k}=1.0$ is the worst. Both NLEVM+PANS $\left(f_{k}=0.5\right)$ and LEVM+PANS $\left(f_{k}=\right.$ 
$0.5)$ does better than their $f_{k}=1.0$ counterparts. However, NLEVM+PANS $\left(f_{k}=0.5\right)$ formulation shows distinct improvement over the LEVM formulation $\left(f_{k}=0.5\right)$ on the rear face (CRD). On face $\mathrm{BC}$, we acknowledge that

the performance of NLEVM+PANS with $f_{k}=0.5$ is actually inferior to LEVM+PANS. On face DTA, performance of the two methodologies seems similar.
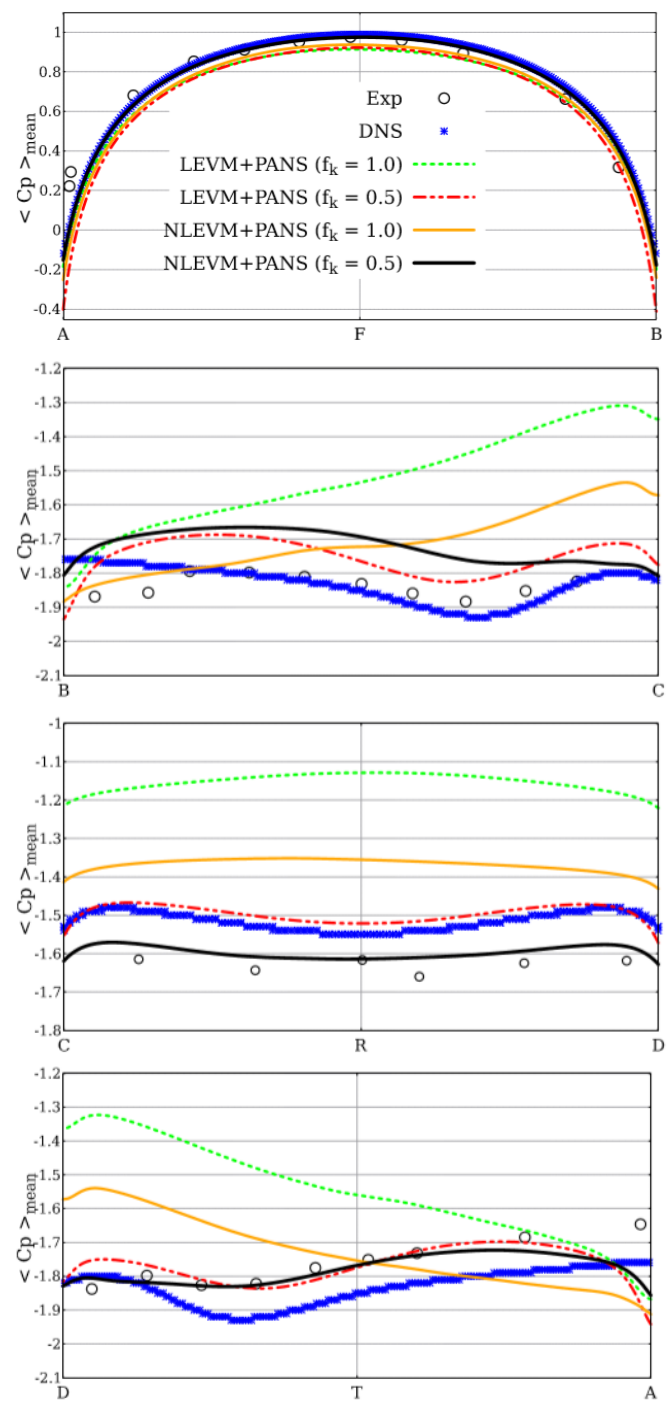

Fig. 6. $\left\langle\mathrm{C}_{\mathrm{p}}\right\rangle_{\text {mean }}$ on cylinder surface: (a) Front face ; (b) Bottom face; (c) Rear face; (d) Top face [top to bottom].

In Fig. 7, we present the mean stream-wise velocity $\left(\left\langle\left\langle\mathrm{V}_{1}\right\rangle\right\rangle_{\text {mean }}\right)$ along the center-line of the cylinder wake. Here, it can be noted that both LEVM+PANS and NLEVM+PANS (with $f_{k}=1$ ) perform poorly in capturing the dip as well as the velocity profile. The NLEVM+PANS methodology does exhibit a clear shift towards the experimental profile as the value of $f_{k}$ is reduced from 1.0 (simulation D) to 0.5 (simulation F). This trend is most evident in the far wake region of $\mathrm{x} / \mathrm{D}>1.5$. For comparison, results from DNS (Trias et al. 2015) and experimental studies (Lyn et al. 1995) are included. Clearly, among our four simulations (A, C, D and F), simulation $\mathrm{F}$ with NLEVM+PANS methodology and $f_{k}=0.5$ provides the best prediction of the center-line wake velocity.

Based on the velocity profiles available in Fig. 7, we have computed the wake recirculation length $\left(\mathrm{Lr}_{\mathrm{r}} / \mathrm{D}\right)$ in each simulation. These results are included in Table 3. The experimental (Lyn et al. 1995) value of the recirculation length is 1.37D. DNS (Trias et al. 2015) report it to around 1.04. In case of $\mathrm{L}_{\mathrm{r}} / \mathrm{D}$, we see clear improvement for LEVM+PANS with reduction in $f_{k}$ value (Table 3 ). However, LEVM+PANS shows considerable error even at $f_{k}$ values of 0.8 and 0.5. NLEVM+PANS $\left(f_{k}=1.0\right)$ - which is essentially non-linear URANS simulation - does better than even LEVM+PANS with $f_{k}=0.5$. While there is a marginal difference between the results of simulations D, E and F (1.35, 1.28 and 1.27 respectively), overall the performance of NLEVM+PANS methodology seems to be superior than LEVM+PANS methodology.

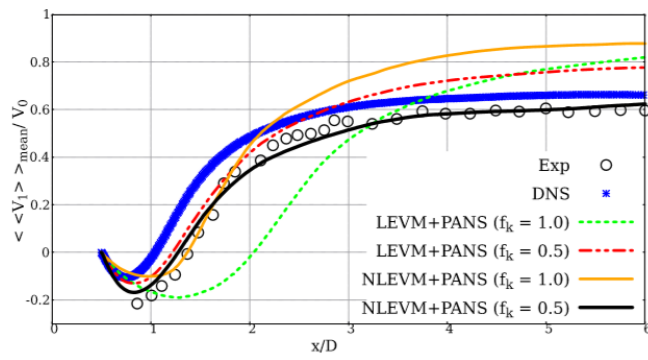

Fig. 7. Mean stream-wise velocity along wake center-line. Exp refers to Lyn et al. (1995). DNS results are of Trias et al. (2015).

Table 3 Comparison of mean quantities

\begin{tabular}{|c|c|c|c|c|}
\hline Case & Simulation & $f_{k}$ & $\left\langle\mathrm{C}_{\mathrm{D}}\right\rangle_{\text {mean }}$ & $\mathrm{L}_{\mathrm{r}} / \mathrm{D}$ \\
\hline A & LEVM+PANS & 1 & 1.76 & 2.03 \\
\hline B & LEVM+PANS & 0.8 & 2.02 & 1.20 \\
\hline C & LEVM+PANS & 0.5 & 2.07 & 1.21 \\
\hline D & NLEVM+PANS & 1 & 1.98 & 1.35 \\
\hline E & NLEVM+PANS & 0.8 & 2.08 & 1.28 \\
\hline F & NLEVM+PANS & 0.5 & 2.25 & 1.27 \\
\hline G & NLEVM+PANS+SR & 0.5 & 2.25 & 1.27 \\
\hline & $\begin{array}{c}\text { Experiment(Lyn } \text { et } \\
\text { al. 1995) }\end{array}$ & - & 2.11 & 1.37 \\
\hline & $\begin{array}{c}\text { DNS (Trias } \text { et al. } \\
\text { 2015) }\end{array}$ & - & 2.18 & 1.04 \\
\hline
\end{tabular}

In Fig. 8 , variation of $\left\langle\mathrm{C}_{\mathrm{P}}\right\rangle_{\text {mean }}$ along the wake center-line behind the square cylinder is presented. Experimental data reported by Nakamura and Ohya (1984) is used as reference besides that of DNS (Trias et al. 2015). Here, LEVM+PANS with $f_{k}=1.0$ fails to capture the $\left\langle\mathrm{C}_{\mathrm{P}}\right\rangle_{\text {mean }}$ wake center-line profile. Though NLEVM+PANS with $f_{k}=1.0$ does slightly better, but it is also too far off from the experimental/DNS profile. There is significant improvement in performance of both LEVM+PANS and NLEVM+PANS as the filter cut-off value is reduced to 0.5 . Among these two, NLEVM+PANS 
( $f_{k}=0.5$ ) does better than LEVM+PANS $\left(f_{k}=0.5\right.$ ) and predict the wake center-line $\left\langle\mathrm{C}_{\mathrm{P}}\right\rangle_{\text {mean }}$ trend close to the one reported by experimental works (Nakamura and Ohya 1984) especially in wake after $\mathrm{x}>1.5$.

We subject the NLEVM+PANS and the LEVM+PANS methodologies to further comparisons in terms of second-order statistics of the velocity field. In Fig. 9 we present $\left(\left(u^{\prime} u^{\prime}\right)^{1 / 2} / V_{0}\right),\left(\left(v^{\prime} v^{\prime}\right)^{1 / 2} / V_{0}\right)$ and $\left(\left(w^{\prime} w^{\prime}\right)^{1 / 2} / V_{0}\right)$ along the wake centerline. These results are compared against the fluctuations observed experimentally by Lyn et al. (1995). We present these results following the precedence of Jeong and Girimaji (2010) and Ranjan and Dewan (2015), who perform similar direct comparisons of the fluctuating moments obtained from PANS simulations against those obtained in experiments. DNS results reported by Trias et al. (2015) are also presented for computational perspective. Evidently, the performance of NLEVM+PANS at both $f_{k}$ values of 1.0 and 0.5 is superior than the corresponding results of LEVM+PANS.

\subsection{Heat Transfer Characteristics}

In context of heat transfer, the most important quantity to focus-on is the non-dimensional mean heat transfer rate (mean Nusselt number) on the cylinder surfaces. In Fig. 10 we present the variation of span-wise averaged $<\mathrm{Nu}\rangle_{\text {mean }}$ on the cylinder surface. Segments AFB, BC, CRD and DTA form this curve (schematic shown in Fig. 1). Since the experimental data is not available at $R_{D}$ of 21,400 , for our comparisons we have included interpolated data at 21,400 using the measured values at $\mathrm{Re}_{\mathrm{D}}$ of 29,600 and 18,500 (Igarashi 1985). Since the measured Nusselt number does show a clear monotonically increasing trend (see Fig. 4a of Igarashi (1985)) with Reynolds number, the authors deem reasonable considering the interpolated values for comparisons

We observe that on segment AFB, all simulations have identical predictions. At the center of the front face (stagnation point F), the mean Nusselt number values predicted by all simulations almost match the experimentally measured value. As one moves toward the cylinder corners $\mathrm{A}$ and $\mathrm{B}$, all simulations tend to over-estimate the heat transfer rate. It is plausible to attribute these overestimates to the the fact that all PANS simulations assume the flow to be turbulent through-out, whereas experiments may have laminar flow in the upstream region. Over the lateral faces (BC and DTA), LEVM+PANS $\left(f_{k}=0.5\right)$ seems slightly inferior to NLEVM+PANS $\left(f_{k}=0.5\right)$. Near point $C$ at bottom face and near point $D$ on top face, the performance of the NLEVM+PANS methodology shows improvement over the corresponding LEVM+PANS cases, especially at $f_{k}=0.5$. Indeed this is the region where shear layer detaches and vortex shedding initiates. On surface CRD (the rear face of the cylinder), the improvement achieved by NLEVM+PANS is most distinct. On this face, it is evident that while
LEVM+PANS shows less sensitivity to a reduced $f_{k}$ value, the NLEVM+PANS meets the expectations of the PANS framework by improving the predictions as the $f_{k}$ value is reduced from 1.0 to 0.5 .

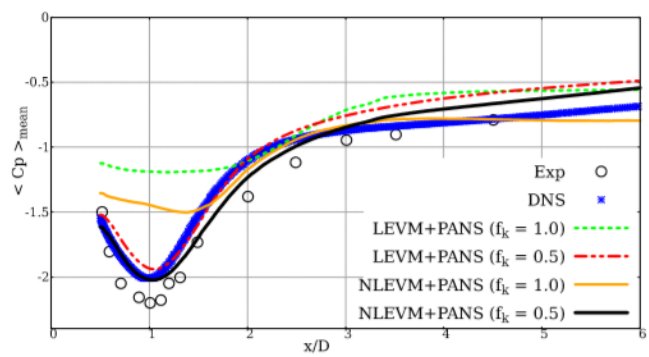

Fig. 8. $\left\langle\mathrm{C}_{\mathrm{p}}>_{\text {mean }}\right.$ value along wake center-line. Exp refers to Nakamura and Ohya (1984).
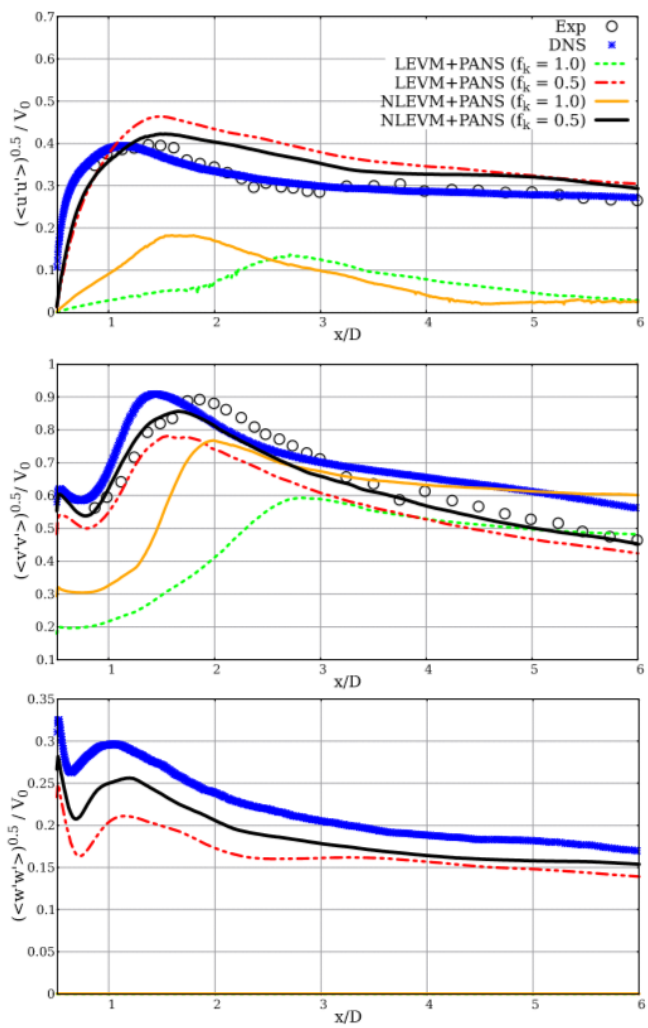

Fig. 9. $\left(\left(u^{\prime} u^{\prime}\right)^{1 / 2} / V_{0}\right)$ (top), $\left(\left(v^{\prime} v^{\prime}\right)^{1 / 2} / V_{0}\right)$ (middle) and $\left(\left(w^{\prime} w^{\prime}\right)^{1 / 2} / V_{0}\right)$ (bottom) variation along the center-line in the wake of the cylinder. Exp refers to Lyn et al. (1995). DNS results are of Trias et al. (2015).

\subsection{NLEVM+PANS+SR vs. NLEVM+PANS}

In Fig. 10, we have presented comparison between the results of NLEVM+PANS with $f_{k}=0.5$ (simulation F) and NLEVM+PANS+SR with $f_{k}=0.5$ (simulation G) methodologies as well. Both these simulations use $f_{k}=0.5$ (see Table 2) and differ only in ways of modeling the thermal flux term. We observe that on front face AFB, the SR-based model does not show any sufficient change in the prediction of mean Nusselt number. However, on all other surfaces indeed some improvements are 
observed in NLEVM+PANS + SR simulation as com-pared to the NLEVM+PANS simulation. Results of simulation $\mathrm{G}$ are closer to the experimental results as compared to the results from simulation E. Even though these improvements are not very substantial in terms of magnitude, still the authors deem the overall trend shown by the NLEVM+PANS+SR methodology to be encouraging.
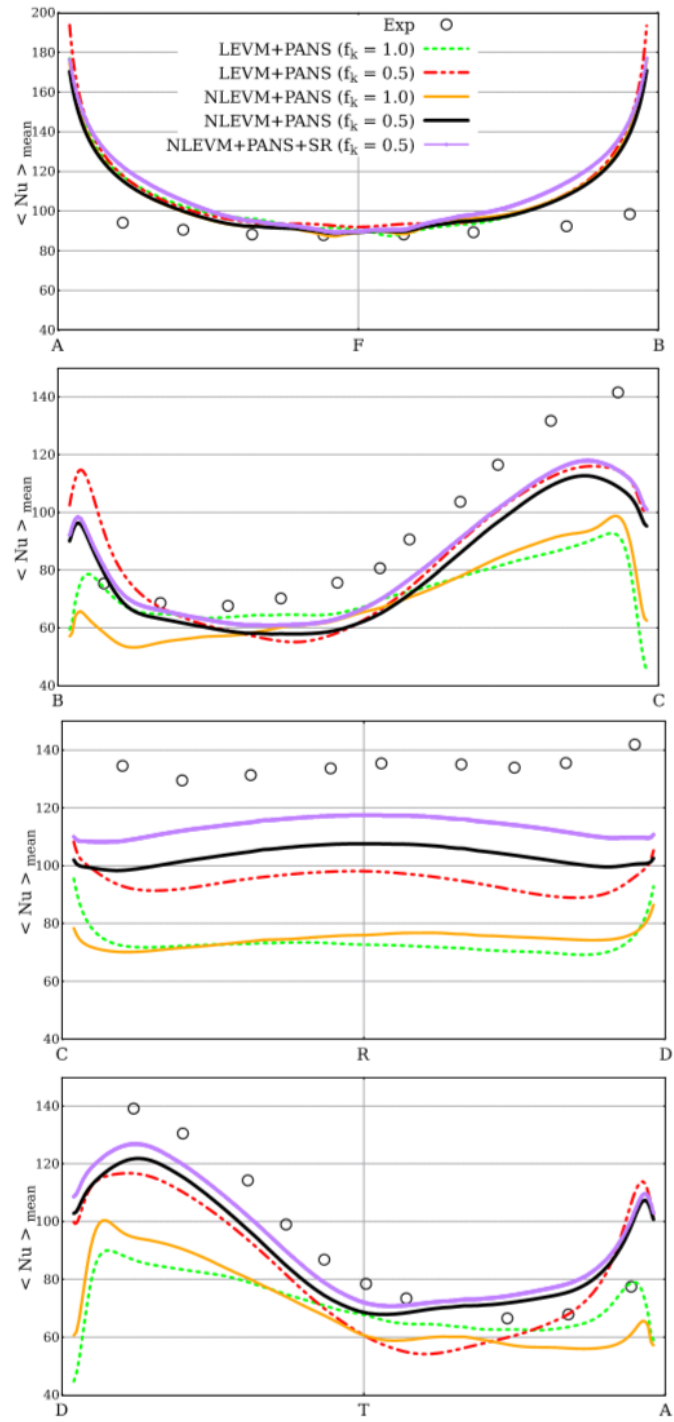

Fig. 10. Variation of $<\mathrm{Nu}>$ mean on the cylinder:(a) Front face; (b) Bottom face; (c) Rear face; (d) Top face [top to bottom].

\subsection{Further Analysis and Explanations}

The PANS methodology is expected to release more scales of motion, when the filter parameter $f_{k}$ is set to have a lower value. A reduction in $f_{k}$ is expected to reduce the level of unresolved kinetic energy $\left(\mathrm{k}_{\mathrm{u}}\right)$, which in turn should reduce the unresolved eddy viscosity, $v_{u}$ (see Eq. 5). A reduced value of $v_{u}$ should then reduce the strength of the modeled turbulent stresses (4) in the filtered momentum equation. This reduction in turbulent stresses is expected to reduce the effective turbulent eddy-diffusion process in the filtered momentum equation, allowing the advection process to release more scales of motion in the instantaneous filtered flow field. As more scales are released, the prediction of flow statistics is expected to show improvement over the corresponding RANS simulation. This is the mechanism of the classical LEVM+PANS methodology to realize improved predictions as compared to the RANS methodology.

In the NLEVM+PANS and the NLEVM + PANS+SR methodologies that we have employed in this work using quadratic model of Shih et al. (1993), a similar mechanism is expected to improve the prediction of flow statistics. However, the important difference between the NLEVM+PANS+SR (or the NLEVM+PANS) and the classical LEVM+PANS methodology is the fact that, in the former, the net diffusive effect in the filtered momentum equation is now determined by both the linear and the nonlinear parts of the turbulent stresses (8). Similarly, the diffusive effect in the filtered temperature equation is determined by the ratio $v_{u} / v$ (in LEVM+PANS and NLEVM+PANS methodologies) and by the stress ratio $(\Gamma)$ in the NLEVM+PANS+SR methodology.

We begin our analysis of the instantaneous flow fields with a general visualization of the flow fields. In Fig. 11 we present contours of the z-component of the filtered vorticity vector. As expected, several vortex cores can be observed as one moves along the wake.

To gauge the extent of smaller scales of motion in the instantaneous filtered fields, we compute the magnitude $(\mathrm{M})$ of the filtered velocity gradient in the flow field. The quantity $M$ is defined as: $M=\sqrt{\frac{\partial\left\langle V_{i}\right\rangle}{\partial x_{j}} \frac{\partial\left\langle V_{i}\right\rangle}{\partial x_{j}}}$. As more scales of motion are released in the flow field, we expect the quantity $\mathrm{M}$ to be more amplified. In Fig. 12, we show contours of $\mathrm{M}$ in the instantaneous flow fields of simulations A-F. We observe that, in general, simulation $\mathrm{C} \& \mathrm{~F}$ (with $f_{k}=0.5$ ) has larger magnitudes of $\mathrm{M}$ compared to simulations A \& D ( $f_{k}=1.0$ ). It clearly suggests that the LEVM+PANS and NLEVM+PANS simulation with $f_{k}=0.5$ (simulation $\mathrm{C} \& \mathrm{~F}$ ) release comparatively more scales of motion than simulations A and D.

To gain a better insight into the wake vortical structures, iso-surfaces of Q-criterion are presented in Fig. 13. Here, the three-dimensional nature of flow field (as corroborated in experimental and computational studies (Trias et al. 2015; Cao and Tamura 2016) is captured by simulations with $f_{k}$ values of 0.5 only. At $f_{k}$ value of unity, both (LEVM and NLEVM) PANS is able to liberate only 2-D scales - which is the familiar expected behaviour of URANS simulation. However, at $f_{k}=0.5$, three dimensional features are evident in the instantaneous flow fields. 
S. Saroha et al. / JAFM, Vol. 13, No. 5, pp. 1453-1469, 2020.

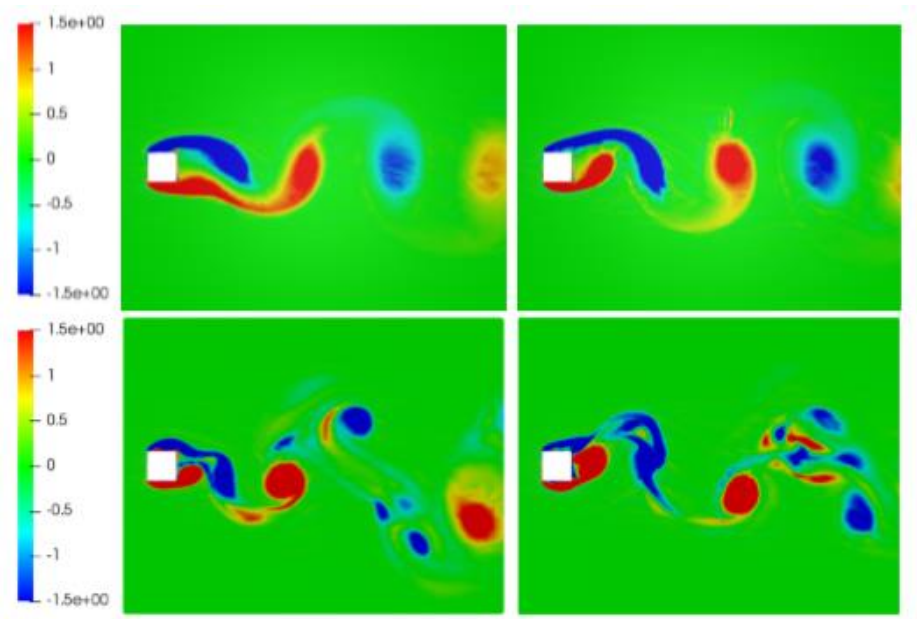

Fig. 11. Instantaneous contours of z-vorticity: (a) LEVM+PANS $\left[f_{k}=1.0\right]$, (b) NLEVM+PANS $\left[f_{k}=1.0\right]$ (top row, left to right); (c) LEVM+PANS [ $\left.f_{k}=0.2\right]$, (d) NLEVM+PANS [ $\left.f_{k}=0.2\right]$ (bottom row, left to right).

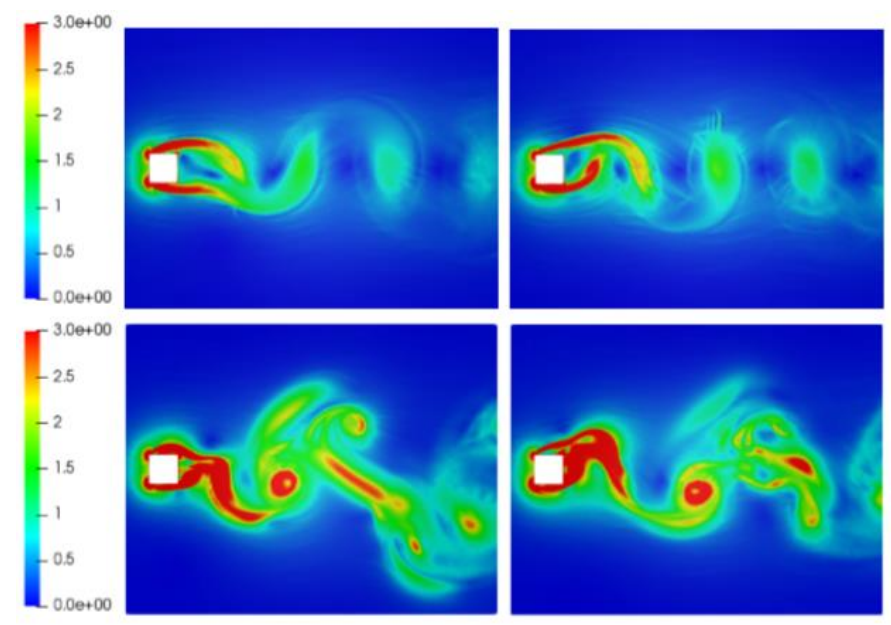

Fig. 12. Instantaneous M-contours: (a) LEVM+PANS $\left[f_{k}=1.0\right]$, (b) NLEVM+PANS $\left[f_{k}=1.0\right]$ (top row, left to right); (c) LEVM+PANS [ $\left.f_{k}=0.2\right]$, (d) NLEVM+PANS [ $\left.f_{k}=0.2\right]$ (bottom row, left to right).

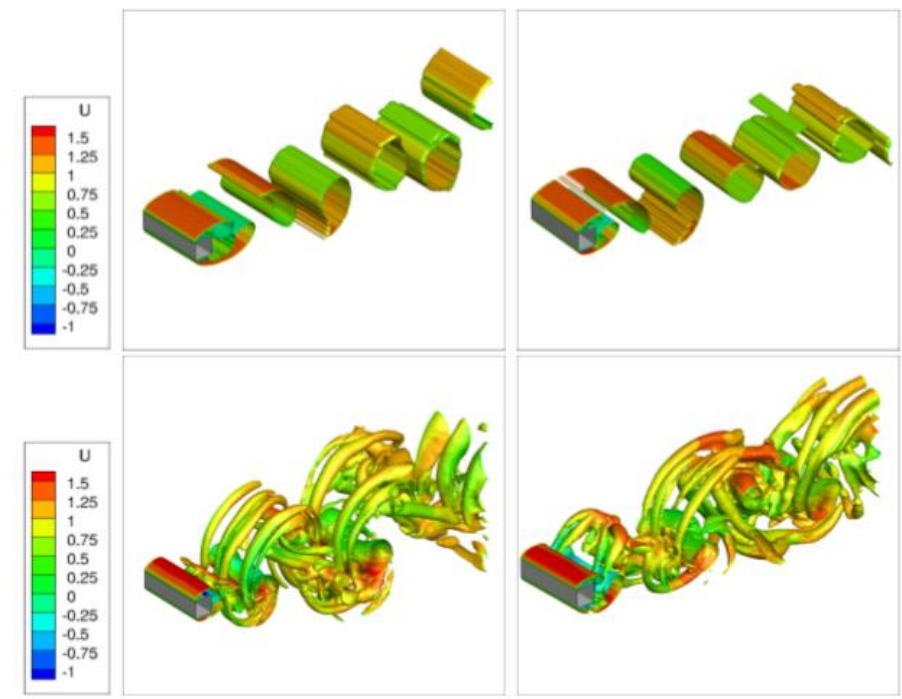

Fig. 13. Iso-surface of Q-criterion: (a) LEVM+PANS $\left[f_{k}=1.0\right]$, (b) NLEVM+PANS $\left[f_{k}=1.0\right]$ (top row, left to right); (c) LEVM+PANS $\left[f_{k}=0.2\right]$, (d) NLEVM+PANS $\left[f_{k}=0.2\right]$ (bottom row, left to right). 

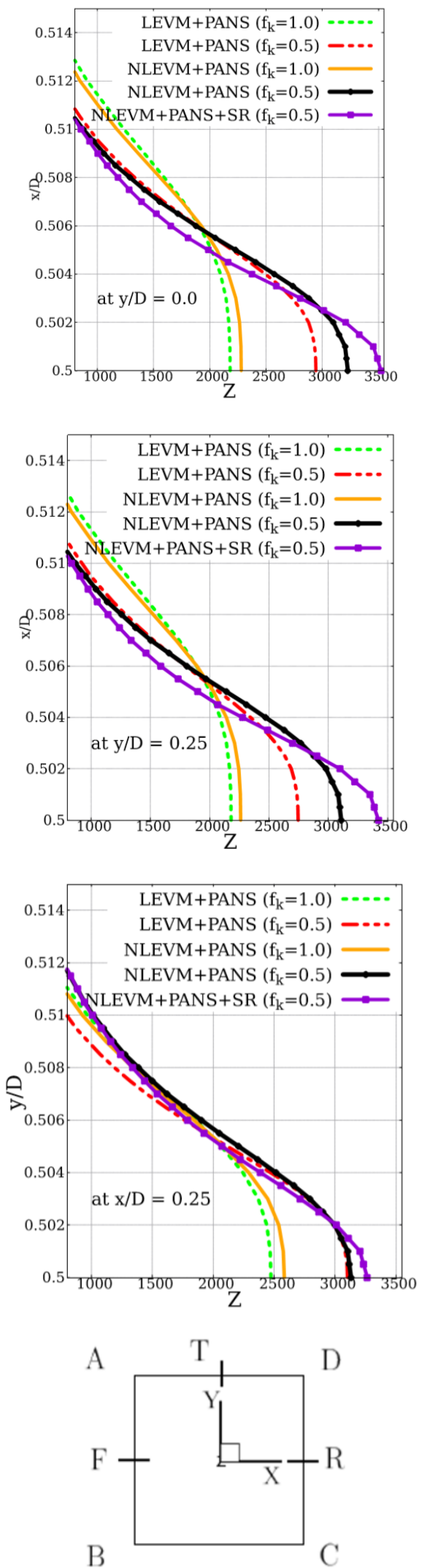

Fig. 14. Variation of $Z$ along wall normal direction.

In line with analysis presented earlier (Fig. 10), we expect the temperature gradient fields also to be more intense in simulations $\mathrm{C}$ (LEVM+PANS $\left.f_{k}=0.5\right)$ and $\mathrm{F}\left(\mathrm{NLEVM}+\mathrm{PANS} f_{k}=0.5\right)$ as compared to those in simulation A, B, D and E. In Fig. 14 we present variation of the magnitude of the gradient of the span-wise averaged mean temperature field (Z) in regions close to faces DTA (top face) and CRD (rear face) of the cylin-der. A quantity $\mathrm{Z}$ is defined as: $Z=\sqrt{\frac{\partial\langle T\rangle}{\partial x_{j}} \frac{\partial\langle T\rangle}{\partial x_{j}}}$. In each of these figures, $Z$ is plotted along the wall-normal direction starting at three specific locations on the cylinder surface. Figure 14a shows variation in $\mathrm{Z}$ along the $\mathrm{x}$-axis at location $y / D=0$ (face CRD). Figure $14 b$ shows variation in $\mathrm{Z}$ along the $\mathrm{x}$-axis at location $\mathrm{y} / \mathrm{D}=$ 0.25 (face CRD). Figure $14 \mathrm{c}$ shows variation in $\mathrm{Z}$ versus the $y$-axis at location $x / D=0.25$ on top face DTS of the cylinder. We observe that at each location, in the near wall regions, NLEVM+PANS+SR (simulation G) is indeed associated with the highest magnitudes of the temperature gradient in the near wall region, suggesting the presence of more small scales in the temperature field in comparisons to other simulations. The highest magnitude in NLEVM+PANS+SR is followed next by those of NLEVM+PANS (simulation F), which in turn, shows higher magnitude of $\mathrm{Z}$ than LEVM+PANS (simulation $\mathrm{C}$ ) in the near-wall regions at the same level of $f_{k}$ value $(=0.5)$. Simulation with higher $f_{k}$ value, like LEVM+PANS and NLEVM+PANS with $f_{k}=1.0$, exhibit lesser magnitude of temperature gradient in the near wall region, thus underpredicting the Nusselt number and near wall thermal fluxes.

In Table 4, we present comparison of the clock time $\left(\tau_{\text {clock }}\right)$ required to simulate one flow-through cycle of various cases utilizing 96 processors. Presented comparison is performed on Intel Haswell CPUs $(2 \times 12$ core, $2.5 \mathrm{GHz})$ E5-2680 node cluster. Conventional lin-ear PANS cases require less clock time of around 12.5 hours to complete one flowthrough cycle. Non-linear PANS cases takes almost $12 \%$ more clock time (14 hours approximately). This must be due to additional non-linear terms in the turbulent stress tensor.

Table 4 Comparison of computational time

\begin{tabular}{|c|c|c|c|}
\hline Case & Simulation & $f_{k}$ & $\begin{array}{c}\tau_{\text {clock }} \\
\text { [in hours] }\end{array}$ \\
\hline A & LEVM+PANS & 1 & 12.5 \\
\hline B & LEVM+PANS & 0.8 & 12.5 \\
\hline C & LEVM+PANS & 0.5 & 12.5 \\
\hline D & NLEVM+PANS & 1 & 14 \\
\hline E & NLEVM+PANS & 0.8 & 14 \\
\hline F & NLEVM+PANS & 0.5 & 14 \\
\hline
\end{tabular}

\section{CONCLUSIONS}

In recent years, the Partially-averaged NavierStokes (PANS) methodology of turbulence computations has emerged as a useful scaleresolving, accuracy-on-demand method. Like the 
Reynolds-averaged Navier-Stokes (RANS) methodology, PANS too relies on turbulence models for the unclosed turbulent stresses in the momentum equation and the unclosed thermal flux in the temperature equation.

In literature, almost all previous implementations and testing of PANS methodology have been done in conjugation with the linear eddy viscosity model for the turbulent stresses and a corresponding linear eddy viscosity- based gradient diffusion hypothesis for the turbulent thermal flux. Thus, the performance of PANS has been naturally restricted by the limitations of these linear closure models. In this work we explore the possibility of further improving the capability of the PANS methodology in simulating flow fields by including the effects of non-linear constitutive equations.

We first extract the PANS version of the non-linear eddy viscosity model of Shih et al. (1993), which was originally proposed as a Reynolds-averaged Navier-Stokes (RANS) closure. Subsequently, we model the thermal flux process using the gradient diffusion hypothesis wherein the diffusion coefficient is modified and sensitized to the presence of non-linear stresses. The resulting nonlinear PANS formulation is evaluated by simulating flow past a heated square cylinder at Reynolds number of 21,400 . To clearly compare and identify the advantages/disadvantages of the non-linear PANS methodology, simulations are performed with the conventional linear PANS methodology as well.

The major conclusions of this study are:

1. Performance of the PANS methodology in conjunction with the non-linear eddy viscosity model of Shih is superior than that of the PANS methodology using with the conventional Boussinesq's linear eddy viscosity model in terms of hydrodynamics quantities (drag, pressure coefficient, velocity profiles and higher order statistics) over the range of filter parameters considered in this work.

2. On the front side of the cylinder, the performance of the non-linear and the linear PANS methodologies are identical in terms of the mean heat transfer rate with both formulations showing over estimations in near edge zones. It is plausible to attribute these overestimates to the fact that the PANS simulations assume fully turbulent flow fields, while in experiments this region may have laminar flow.

3. On the rear side of the cylinder and over the aft region of the lateral wall (where shear detachment takes place and vortex shedding initiates), the non-linear PANS formulation shows some improvements over the linear PANS formulation. This improvement is further enhanced (on the lateral and the rear cylinder surfaces) in the simulation where the diffusivity of the modeled thermal flux process is directly sensitized to non-linear stresses in the flow field (the SR version of NLEVM+PANS methodology). Even though these improvements are not very substantial magnitude wise, the qualitative trend shown by the non-linear PANS methodology is encouraging.

Based on the evaluations presented in this study, the authors conclude that the non-linear PANS methodology seems to have an inherent potential to improve the performance of PANS over the conventional its linear counterpart on the same given grid and identical filter settings. There is a need to perform more such comparative studies (with more test cases and with more non-linear eddy viscosity models) to fully examine and leverage this potential for further improving PANS predictions of turbulent flow fields. Indeed, it is pertinent to mention that we have performed similar evaluations for another canonical flow field: flow past a sphere. For sphere too, the performance of the NLEVM+PANS methodology is found to be superior than its LEVM+PANS counterpart. Due to page limitations, we have not been able to include those results here. Those comparisons will appear in detail in our next manuscript.

\section{ACKNOWLEDGMENTS}

The authors thank IIT Delhi HPC facility for computational resources.

\section{REFERENCES}

Abdol-Hamid, K. S. and S. S. Girimaji (2004). A two-stage procedure toward the efficient implementation of pans and other hybrid turbulence models. NASA Technical Memorandum 213260.

Abe, K., T. Kondoh and Y. Nagano (1994). A new turbulence model for predicting fluid flow and heat transfer in separating and reattaching flows-i. flow field calculations. International Journal of Heat and Mass Transfer 37(1), 139-151.

Abe, K., T. Kondoh and Y. Nagano (1995). A new turbulence model for predicting fluid flow and heat transfer in separating and reattaching flows-ii. thermal field calculations. International Journal of Heat and Mass Transfer 38(8), 1467-1481.

Apsley, D., W. Chen, M. Leschziner and F. Lien (1997). Non-linear eddy-viscosity modelling of separated flows. Journal of Hydraulic Research 35(6), 723-748.

Argyropoulos, C. and N. Markatos (2015). Recent advances on the numerical modelling of turbulent flows. Applied Mathematical Modelling 39(2), 693-732.

Aus der Wiesche, S. (2007). Large-eddy simulation study of an air flow past a heated square 
cylinder. Heat and mass Transfer 43(6), 515525.

Ayyappan, T. and S. Vengadesan (2008). Threedimensional simulation of flow past a circular cylinder by nonlinear turbulence model. Numerical Heat Transfer, Part A: Applications 54(2), 221-234.

Basara, B., P. Z. and S. Girimaji (2018). A new approach for the calculation of the cut-off resolution parameter in bridging methods for turbulent flow simulation. International Journal of Heat and Fluid Flow 74, 76-78.

Boussinesq, J. (1877). Essay on the Theory of Running Waters. Imprimerie Nationale.

Cao, Y. and T. Tamura (2016). large-eddy simulations of flow past a square cylinder using structured and unstructured grids. Computers and Fluids 137, 36-54.

Chen, H. and V. Patel (1988). Near-wall turbulence models for complex flows including separation. AIAA Journal 26(6), 641-648.

Chesnakas, C. J., D. Taylor and R. L. Simpson (1997). Detailed investigation of the threedimensional separation about a 6: 1 prolate spheroid. AIAA Journal 35(6), 990- 999.

Colombo, E., F. Inzoli and R. Mereu (2008). Capability of non-liner eddy viscosity model in predicting complex flows. HEFAT 2008.

Craft, T., B. Launder and K. Suga (1996). Development and application of a cubic eddyviscosity model of turbulence. International Journal of Heat and Fluid Flow 17(2), 108115.

Daly, B. J. and F. H. Harlow (1970). Transport equations in turbulence. The Physics of Fluids 13(11), 2634-2649.

Franke, R. and W. Rodi (1993). Calculation of vortex shedding past a square cylinder with various turbulence models. In Turbulent Shear Flows 8, 189-204. Springer.

Frendi, A., A. Tosh and S. Girimaji (2006). Flow past a backward-facing step: comparison of pans, des and urans results with experiments. International Journal for Computational Methods in Engineering Science and Mechanics 8(1), 23-38.

Gatski, T. and T. Jongen (2000). Nonlinear eddy viscosity and algebraic stress models for solving complex turbulent flows. Progress in Aerospace Sciences 36(8), 655-682.

Germano, M. (1992). Turbulence: the filtering approach. Journal of Fluid Mechanics 238, 325-336.

Girimaji, S. S. (1997). A galilean invariant explicit algebraic reynolds stress model for turbulent curved flows. Physics of Fluids 9(4), 10671077 .
Girimaji, S. S. (2006). Partially-averaged navierstokes model for turbulence: A reynoldsaveraged navierstokes to direct numerical simulation bridging method. Journal of Applied Mechanics 73(3), 413-421.

Hellsten, A. and S. Wallin (2009). Explicit algebraic reynolds stress and non-linear eddy-viscosity models. International Journal of Computational Fluid Dynamics 23(4), 349361.

Igarashi, T. (1984). Characteristics of the flow around a square prism. Bulletin of JSME 27(231), 1858-1865.

Igarashi, T. (1985). Heat transfer from a square prism to an air stream. International Journal of Heat and Mass Transfer 28(1), 175-181.

Ishihara, T. and K. Hibi (2002). Numerical study of turbulent wake flow behind a threedimensional steep hill. Wind and Structures 5(2 34$), 317-328$

Jeong, E. and S. Girimaji (2010). Partially averaged navierstokes (pans) method for turbulence simulations - flow past a square cylinder. Journal of Fluids Engineering 132(12), 121203 .

Kimura, I. and T. Hosoda (2003). A non-linear k-E model with realizability for prediction of flows around bluff bodies. International Journal for Numerical Methods in Fluids 42(8), 813-837.

Lakshmipathy, S. and S. Girimaji (2010). Partially averaged navier-stokes (pans) method for turbulence simulations: flow past a circular cylinder. Journal of Fluids Engineering 132(12), 121202

Launder, B. and D. Spalding (1974). The numerical computation of turbulent flows. Computer Methods in Applied Mechanics and Energy 3(2), 269-289.

Liu, Z. (2010). Square cylinder large eddy simulation based on random inlet boundary condition. Journal of Applied Fluid Mechanics 3(1), 35-45.

Lumley, J. (1970). Toward a turbulent constitutive relation. Journal of Fluid Mechanics 41(2), 413-434.

Lyn, D., S. Einav, W. Rodi and J. Park (1995). A laserdoppler velocimetry study of ensembleaveraged characteristics of the turbulent near wake of a square cylinder. Journal of Fluid Mechanics 304, 285-319.

Moulai, S., A. Korichi and G. Polidori (2016). Aided mixed convection past a heated square cylinder at low blockage ratio. Journal of Applied Fluid Mechanics 9(1), 303-310.

Nakamura, Y. and Y. Ohya (1984). The effects of turbulence on the mean flow past twodimensional rectangular cylinders. Journal of Fluid Mechanics 149, 255-273. 
Nakayama, A. and S. Vengadesan (2002). On the influence of numerical schemes and subgridstress models on large eddy simulation of turbulent flow past a square cylinder. International Journal for Numerical Methods in Fluids 38(3), 227-253.

Pereira, F. S., L. Eca, G. Vaz and S. S. Girimaji (2018a). Challenges in scale-resolving simulations of turbulent wake flows with coherent structures. Journal of Computational Physics 363, 98-115.

Pereira, F., G., Vaz, L. Eca and S. Girimaji (2018b). Simulation of the flow around a circular cylinder at re $=3900$ with partially-averaged navier-stokes equations. International Journal of Heat and Fluid Flow 69, 234-246.

Ramesh, V., S. Vengadesan and J. Narasimhan (2006). 3d unsteady rans simulation of turbulent flow over bluff body by non-linear model. International Journal of Numerical Methods for Heat \& Fluid Flow 16(6), 660673.

Ranjan, P. and A. Dewan (2015). Partially averaged navier stokes simulation of turbulent heat transfer from a square cylinder. International Journal of Heat and Mass Transfer 89, 251266.

Rao, A. N., G. Mineli, B. Basara and K. S. (2018). Investigation of the near wake flow topology of a simplified heavy vehicle using pans simulations. Journal of Fluid Engineering and Industrial Aerodynamics 183, 243-272.

Rodi, W. (1997). Comparison of les and rans calculations of the flow around bluff bodies. Journal of Wind Engineering and Industrial Aerodynamics 69, 55-75.

Rubinstein, R. and J. Barton (1990). Nonlinear Reynolds stress models and the renormalization group. Physics of Fluids A: Fluid Dynamics 2(8), 1472-1476.

Saroha, S., S. S. Sinha and S. Lakshmipathy (2018). Evaluation of partially averaged navier-stokes method in simulating flow past a sphere. Journal of Applied Fluid Mechanics 11(5) 1333-1348.

Saroha, S., S. S. Sinha and S. Lakshmipathy (2019). Evaluation of pans method in conjunction with non-linear eddy viscosity closure using openfoam. International Journal of Numerical
Methods for Heat \& Fluid Flow 29(3), 949980.

Schiestel, R. and A. Dejoan (2005). Towards a new partially integrated transport model for coarse grid and unsteady turbulent flow simulations. Theoretical and Computational Fluid Dynamics 18(6), 443-468.

Shih, T., J. Zhu and J. Lumley (1993). A realizable reynolds stress algebraic equation model. NASA Technical Memorandum 105993.

Shih, T. H., J. Zhu, W. Liou, K. H. Chen, N.-S. Liu and J. L. Lumley (1997). Modelling of turbulent swirling flows. NASA TM 113112. ICOMP-97-08, CMOTT-97-03.

Song, C. S. and S. O. Park (2009). Numerical simulation of flow past a square cylinder using partially-averaged navier-stokes model. Journal of Wind Engineering and Industrial Aerodynamics 97(1), 37-47.

Speziale, C. (1987). On nonlinear kl and k- $\varepsilon$ models of turbulence. Journal of Fluid Mechanics 178, 459-475.

Trias, F., A. Gorobets and A. Oliva (2015). Turbulent flow around asquare cylinderat reynoldsnumber22000: adns study. Computers and Fluids 123(-), 87-98.

Wallin, S. and A. V. Johansson (2000). An explicit algebraic reynolds stress model for incompressible and compressible turbulent flows. Journal of Fluid Mechanics 403, 89132.

Wikström, P., S. Wallin and A. V. Johansson (2000). Derivation and investigation of a new explicit algebraic model for the passive scalar flux. Physics of Fluids 12(3), 688-702.

Yang, X., H. Ma and Y. Huang (2005). Prediction of homogeneous shear flow and a backwardfacing step flow with some linear and nonlinear $\mathrm{k}-\varepsilon$ turbulence models. Communications in Nonlinear Science and Numerical Simulation 10(3), 315-328.

Yangaz, M. U., C. G. A. and M. A. N. Kadirgan (2019). Comparison of conventional and modified burners in performance with different fuels using a linear and a nonlinear eddyviscosity turbulence model. Journal of Applied Fluid Mechanics 12(6), 20169-2081. 